\title{
Political differences in free will belief are associated with differences in moralization
}

\author{
Jim Albert Charlton Everett*, Cory J. Clark*2, Peter Meindl3, Jamie B. Luguri4, Brian D. \\ Earp,5, Jesse Graham6, Peter H. Ditto7, \& Azim F. Shariff8 \\ I School of Psychology, University of Kent \\ 2 Department of Psychology, Durham University. \\ 3 Department of Psychology, Calvin College. \\ 4 The Law School, University of Chicago. \\ 5 Department of Psychology, Yale University. \\ 6 Department of Management, University of Utah. \\ 7 Department of Psychological Science, University of California, Irvine. \\ \& Department of Psychology, University of British Columbia.
}

Manuscript Forthcoming in the

Journal of Personality and Social Psychology: Personality Processes and Individual Differences

\begin{abstract}
In fourteen studies, we tested whether political conservatives' stronger free will beliefs were linked to stronger and broader tendencies to moralize, and thus a greater motivation to assign blame. In Study I (meta-analysis of five studies, $n=308,499$ ) we show that conservatives have stronger tendencies to moralize than liberals, even for moralization measures containing zero political content (e.g., moral badness ratings of faces and personality traits). In Study 2, we show that conservatives report higher free will belief, and this is statistically mediated by the belief that people should be held morally responsible for their bad behavior $(n=14,707)$. In Study 3, we show that political conservatism is associated with higher attributions of free will for specific events. Turning to experimental manipulations to test our hypotheses, we show that: when conservatives and liberals see an action as equally wrong there is no difference in free will attributions (Study 4); when conservatives see an action as less wrong than liberals, they attribute less free will (Study 5); and specific perceptions of wrongness account for the relation between political ideology and free will attributions (Study 6a and 6b). Finally, we show that political conservatives and liberals even differentially attribute free will for the same action depending on who performed it (Studies 7a-d). These results are consistent with our theory that political differences in free will belief are at least partly explicable by conservatives' tendency to moralize, which strengthens motivation to justify blame with stronger belief in free will and personal accountability.
\end{abstract}

free will; morality; blame; motivated cognition; political psychology

\section{Author Note}

* Indicates shared first-authorship. Correspondence can be directed at either:

Jim A.C. Everett at Keynes College, Canterbury, Kent or j.a.c.everett@kent.ac.uk or Cory J. Clark at Department of Psychology, Durham or cory.j.clark@durham.ac.uk 
"We must reject the idea that every time a law's broken, society is guilty rather than the lawbreaker. It is time to restore the American precept that each individual is accountable for his actions."

Ronald Reagan (1968)

Liberals and conservatives characteristically view the relationship between the individual and society in different terms. Whereas liberal (i.e., left-wing) ideology has often focused on the role of social institutions and other external forces in shaping individual behavior, conservative (i.e., right wing) thinking tends to emphasize the importance of personal responsibility (Eidelman et al., 2012; Jost et al., 2008; Skitka et al., 2002; Skitka \& Tetlock, 1992, 1993). According to the conservative view, individuals should take responsibility for the course of their own lives and refrain from expecting others to solve their problems. In addition to being explicitly championed by prominent conservative leaders (Cameron, 2010; Reagan, 1968; Thatcher, 1981), a focus on personal responsibility seems to pervade the thinking of everyday conservatives as well (Carey \& Paulhus, 20I3). Research has shown that conservatives are more likely than liberals to make dispositional attributions of responsibility in a number of key areas, including poverty (Zucker \& Weiner, 1993), unemployment (Feather, 1985), obesity (Crandall, 1994), and even intelligence (Skitka et al., 2002).

In addition to judging that others are more responsible for their actions, recent research by Carey and Paulhus (2013) has suggested that conservatives also believe that others have more free will. Political conservatism is not merely associated with thinking that others are more responsible for their specific actions, but also with thinking that they have more autonomous control over their behavior in general. Across three studies, Carey and Paulhus (2013) found that belief in free will was associated with traditional conservative attitudes as well as with an increased importance attached to the three 'conservative' moral foundations (loyalty, authority, sanctity). Why might this be so?

We suggest that the relationship between political orientation and free will belief might be parsimoniously explained by motivated social cognition. This hypothesis is derived from two areas of research. First, recent research has demonstrated that free will beliefs are motivated by desires to punish others (Clark et al., 20l4) and to justify holding them morally responsible (Clark, Baumeister, \& Ditto, 20I7), which recently has been replicated and confirmed in meta-analyses (Clark, Winegard, \& Shariff, 2019). Second, political conservatives have a tendency to moralize a wider scope of actions than their liberal counterparts (Graham et al., 2009, 20II, 2013). Combining these two areas of research, we suggest that conservatives report greater belief in free will and attribute more free will to people than do liberals because conservatives recognize a wider spectrum of transgressions for which moral responsibility must be assigned and moral blame attributed.

\section{Motivated Beliefs in Free Will}

What do we mean by "free will?" In this paper, we draw on an understanding of free will that has both been articulated by philosophers and seems to track the intuitions of laypeople. In line with previous empirical work in this area, we use the term "free will" to refer to an autonomous choice of action that a person performs in the absence of substantial 
internal and external constraints (Baumeister \& Monroe, 20I4; Paulhus \& Carey, 20I I), where this ability to choose renders one morally responsible for their actions (Nichols, 2007; Nichols \& Knobe, 2007). Free will, in other words, can be understood as responsibility-making autonomy. Note that the concept of free will is distinct from the concept of attributions in social psychology (e.g., Skitka et al., 2002; Zucker \& Weiner, 1993), and this can broadly be related to the philosophical distinction between reasons and causes. Attributions are reasons, and help answer the question of what the reason is for why a person performed a given action. In social psychology, work on attribution has focused on two main kinds of reasons: dispositional attributions (the person did it because of the kind of person they are); and situational attributions (the person did it because of the situation they were placed in). In contrast, the concept of free will relates to causes, which can partially include reasons but also ultimate level causal factors (e.g., it was determined by genes). To illustrate: it is perfectly plausible to say that someone stole something because they are a selfish person (a dispositional attribution), but that because their selfishness was genetically determined (an attribution of free will), they did not have free and thus were not personally responsible.

Assuming this definition of free will of responsibility-making autonomy, what would it mean for belief in free will to be "motivated," as we suggested? Motivated social cognition refers to the well-documented tendency for desired conclusions to organize judgment processes in a top-down fashion that favors evidence for the conclusions people prefer (Ditto et al., 2009). When reasoning about the world, people often act more like intuitive lawyers than intuitive scientists, such that their desired beliefs influence their actual beliefs (Baumeister \& Newman, 1994; Haidt, 200I, 2012). In moral reasoning, desires to blame and to hold individuals morally responsible compel people to produce rational explanations that would justify their moral judgments (Alicke, 2000; Clark et al., 20I5). Indeed, a growing body of research has demonstrated that the desire to hold individuals morally accountable for their immoral behaviors can lead to motivated judgments that such immoral behaviors are intended, under the agent's control, and freely chosen (Alicke, 1992, 2000; Alicke, Rose, \& Bloom, 20I I; Clark et al., 20I4; Clark, Bauman, Kamble, \& Knowles, 20I7; Clark, Winegard, \& Baumeister, 2019; Cushman, Knobe, \& Sinnott-Armstrong, 2008; Hamlin \& Baron, 20I4; Knobe, 2003; Knobe \& Fraser, 2008; Leslie, Knobe, \& Cohen, 2006; Phillips \& Knobe, 2009).

But how might belief in free will, specifically, be seen as a form of motivated social cognition? Across five studies, Clark et al. (2014) used a range of methods - experimental, correlational, and archival - to test the hypothesis that a key motivation underlying belief in human free will is the desire to hold others morally responsible for their behavior. For example, telling students that a fellow classmate had cheated on a recent exam increased belief in free will on a standard measure of global free will belief; and countries with higher homicide rates were also found to express higher levels of free will belief. Clark et al (20I4) concluded that free will belief is not an abstract, invariant phenomenon, but is rather linked, at least in part, to a motivated desire to hold others morally responsible for their wrongful behaviors, the strength of which varies across time and situation. 
The focus on wrongful behaviors may have a straightforward explanation. Put simply, across a broad range of psychological phenomena, "bad is stronger than good" (Baumeister et al., 200I, p. I), meaning that people tend to notice, and give greater weight to, negative actions and outcomes than positive ones. For example, research has repeatedly shown a praise-blame asymmetry in judgments of intentional action: people are more inclined to say that a behavior with negative side-effects was performed intentionally than an identical action with positive side-effects (Knobe, 2003; Pettit \& Knobe, 2009). Motivated judgments of others' behavior are most pronounced in - and perhaps even driven by - cases in which the behavior is seen as harmful (Alicke et al., 2008). All else being equal, the desire to blame another for bad behavior is more potent than the desire to praise another for their good behavior (Clark, Shniderman, Baumeister, Luguri, \& Ditto, 2018). As Baumeister et al. (200I) note, while a general explanation for this effect is hard to come by given its inherent generality across a broad range of psychological phenomena, it is likely that a tendency to pay greater attention to bad actions and outcomes than good ones will have been evolutionarily adaptive because survival often requires more urgent attention to possible bad outcomes (e.g., a predator behind you) than possible good outcomes (e.g., a berry bush behind you).

\section{Political Differences in Morality}

What could explain a greater belief in free will among conservatives compared to liberals? It is possible that this difference simply reflects a fundamental underlying political difference in perceptions of how much freedom and control people have over their behavior and life outcomes. But given the work of Clark et al. (20I4) on free will belief being driven by a desire to blame, we predicted that conservatives' greater endorsement of belief in free will might be due-at least in part-to conservatives' stronger tendency to moralize. According to a large body of research, political conservatives and liberals differ in many more ways than their preferred political candidates (Amodio et al., 2007; Jost et al., 2009), including at the cognitive level. Most importantly for the purposes of this paper, a growing body of evidence has demonstrated that political orientations are associated with differences in judgments in the moral domain, both in terms of what constitutes a moral issue in the first place as well as how wrong a particular action/behavior is.

The social intuitionist approach suggests that moral judgment is largely a motivated phenomenon: moral judgment is triggered by quick moral intuitions, and moral reasoning largely serves as a post-hoc rationalization of these intuitive judgments (Haidt, 200 I). Moral Foundations Theory (Graham et al., 20II, 2013) is rooted in work on motivated cognition and the social intuitionist approach to morality (Baumeister \& Newman, 1994; Ditto et al., 2009; Haidt, 200 I, 20I2) and posits that conservatives have a broader moral domain than do liberals. In other words, liberals are less inclined to perceive a variety of actions as morally relevant than conservatives (Graham et al., 2009) - a finding demonstrated by analyzing data drawn from participants from I I different world regions (Graham et al., $20 \mathrm{II}$ ), and from life narrative interviews with politically engaged adults (McAdams et al., 2008). Similarly, we note, political conservatism is associated with more punitiveness in general (e.g., Carroll et al., 1987; Sargent, 2004). So while liberals can and do find some actions more morally wrong than conservatives (Frimer et al., 2017), the available evidence 
does suggest that conservatives tend to view a broader range of actions as having moral significance,, and therefore as more suitable for moral judgment and blame. We demonstrate this ourselves in Study I of the manuscript, using a meta-analysis of five new studies with a combined $n$ of 308,499 , establishing the conservative tendency to moralize.

\section{Practical Significance}

If our hypothesis is correct, this could help explain a variety of political disagreements between liberals and conservatives regarding the degree to which various groups (e.g., the unemployed, the homeless, prisoners, women with unwanted pregnancies, etc.) are responsible for their plights, and thus how they should be treated by governmental policies. Moreover, our hypothesized results might help explain why these kinds of disagreements seem so intractable. If policy differences result from affect-based moral intuitions about responsibility - and liberals and conservatives have different moral intuitions - it may be difficult or impossible for liberals and conservatives to agree on the "correct" policy solutions. And these disagreements should not be expected to be solved by collecting more and better data.

\section{The Present Research}

We conducted a series of I4 studies to explore this motivated cognition account whereby conservatives ascribe more free will because they have a broader moral domain, and thus more often perceive actions as appropriately subject to moral judgment. In other words, we predicted that because liberals think that a narrower range of phenomena constitute moral issues, they have a narrower range of actions for which judgments of blame and attributions of free will are deemed to be appropriate. If this prediction is correct, I) higher tendencies to blame should account for conservatives' stronger belief in free will, and 2) political liberals and conservatives should differ in attributions of free will only when there are corresponding differences in the extent to which they perceive actions to be morally wrong. In short, we should find that conservatives believe more in free will and ascribe more free will generally (Studies 1-3), but in instances where differences in perceived moral wrongness can be removed or reversed, differences in free will ascriptions should similarly be absent or in the opposite direction, respectively (Studies 4-7). Experimental materials, pre-registrations, data, analysis code, and results can be seen at the Open Science Framework: https://osf.io/ny82c/

\section{Meta-analysis of Studies I a-Ie}

This opening meta-analysis was conducted to establish the relationship between more conservative political ideology and moralization. We combined data from five studies drawn from a variety of populations with a total $n$ of 308,499. Each study included a measure of political ideology and one or more measures of moralization.

\footnotetext{
I While aspects of our argument are adjacent to Moral Foundations Theory (MFT), we do not depend on the specifics of MFT and will avoid these arguments in this paper. We believe the findings from MFT support our assertion that conservatives moralize more than liberals, but we do not rely exclusively on MFT to make this point. We establish this ourselves in Study I. Moreover, we take no stance on the kinds or categories of moral intuitions that liberals or conservatives might have, nor whether these kinds or categories can all fit under a "harm" umbrella or whether they reflect distinct moral modules.
} 


\section{Studies Ia-I e Methods}

Table I contains summary details (sample information, ideology and moralization measures) for Studies Ia-le and full details of all study methods are available at the OSF.

Ethics Statement. Studies Ia and Ic received ethical approval from the University of Southern California's Institutional Review Board (“Morality Studies”; UP-07-00393). Studies Id and Ie were covered by a separate application from the same institution ("Reading, Thoughts, and Behavior"; UP-I2-00388). In Study Ib, we conducted secondary data analysis of a publicly available dataset provided by the "Measuring Morality" project based at Duke University.

Participants. Studies Ia and Ic were conducted on yourmorals.org, a survey website on which participants (mostly from the U.S., but some from across the globe) complete surveys in exchange for response feedback. For Study Ib, data were drawn from the Measuring Morality Survey from a nationally representative panel of adult participants maintained by Knowledge Networks. Studies Id and le were conducted on Amazon Mechanical Turk (Mturk) with U.S. participants. Mturk is not perfectly representative of the U.S. as a whole, but it is diverse with respect to age, sex, race, education, SES, and ideology - more so than student samples are (Paolacci \& Chandler, 20I4).

Ideology. Ideology was self-reported on 7-point scales in all studies. In Studies Ia, Ic, and Id, the poles were "Very liberal" to "Very conservative." In Study Ib, these were "Extremely liberal" to "Extremely conservative." In Study le, participants self-reported their political ideology on economic issues, social issues, foreign policy issues, and overall on the same scale as Study Ib, which were combined into an index of ideology.

Moralization. Full details of each moralization measure are available at the OSF. Some of the moralization measures can be criticized for containing politically relevant items (e.g., religious items), and so the meta-analysis was conducted twice, once with all moralization measures, and once with the politically irrelevant measures only. The measures that were excluded in the politically irrelevant version of the meta-analysis have an asterisk by their name in the descriptions below and in Table I.

In Study Ia, moralization was measured using The Moral Foundations Questionnaire* (MFQ; Graham et al., 2009). Study Ib included three moralization measures: (I) The Moralization of Everyday Life Scale (MELS; Lovett et al., 20I2), (2) The Moral Foundations Sacredness Scale* (MFSS; Graham \& Haidt, 20I2), and (3) The Ethical Values Assessment* (EVA; Padilla-Walker \& Jensen, 2016).

In Study Ic, moralization was measured by having participants evaluate how important it was for a person to possess various characteristics in order to be a morally good person. Participants completed a random subset of 45-47 characteristics from a list of 92 . This list was created by combining previous studies that attempted to create representative lists of moralized characteristics (Aquino \& Reed II, 2002; Cawley et al., 2000; Lapsley \& Lasky, 200 I; K. D. Smith et al., 2007; Walker \& Pitts, 1998) and conducting a large scale pretest in which 4,565 yourmorals.org participants were asked to report a) their most important moral values and b) behaviors for which they most often morally judged people.

Study Id measured moralization by having participants view 16 pictures of faces and rate how morally bad the depicted person probably was. The 16 pictures were taken from a 
larger set of pictures shown to effectively capture four main quadrants of social perception (e.g. Harris \& Fiske, 2006): Warmth/Incompetence; Coldness/Incompetence; Coldness/Competence; Warmth/Competence. Study le measured moralization by having participants rate how morally bad 30 personality traits are. These 30 traits were the individual personality facets from the NEO PI-R (Costa \& McCrae, I992a, 1992b).

\section{Meta-analysis Methods}

We employed many of the suggested procedures outlined by Goh, Hall, and Rosenthal (2016) to conduct our meta-analysis. Because the studies were drawn from different populations and used different measures of moralization, we report random effects (Goh et al., 2016; see also Hedges \& Vevea, 1998). We included one effect size for each study (i.e., the effect sizes for the three moralization measures in Study Ib were averaged; Card, 20I2), thus five $r$ effect sizes (the correlations between more conservative ideology and higher moralization) were included. We conducted one-sample $t$-tests of the effect sizes first with all moralization measures and then with the potentially politically confounded ones removed.

\section{Results}

As can be seen in summary Table I, more conservative political ideology was positively and significantly related to greater moralization in every single study ( $p s<.027$ ), including those containing zero political content. The meta-analysis revealed a small to medium effect size overall, $r=.27, p=.003$; which remained small to medium and significant with the politically irrelevant measures only, $r=.24, p=.012$. 
Table I

Moralization meta-analysis summary and results

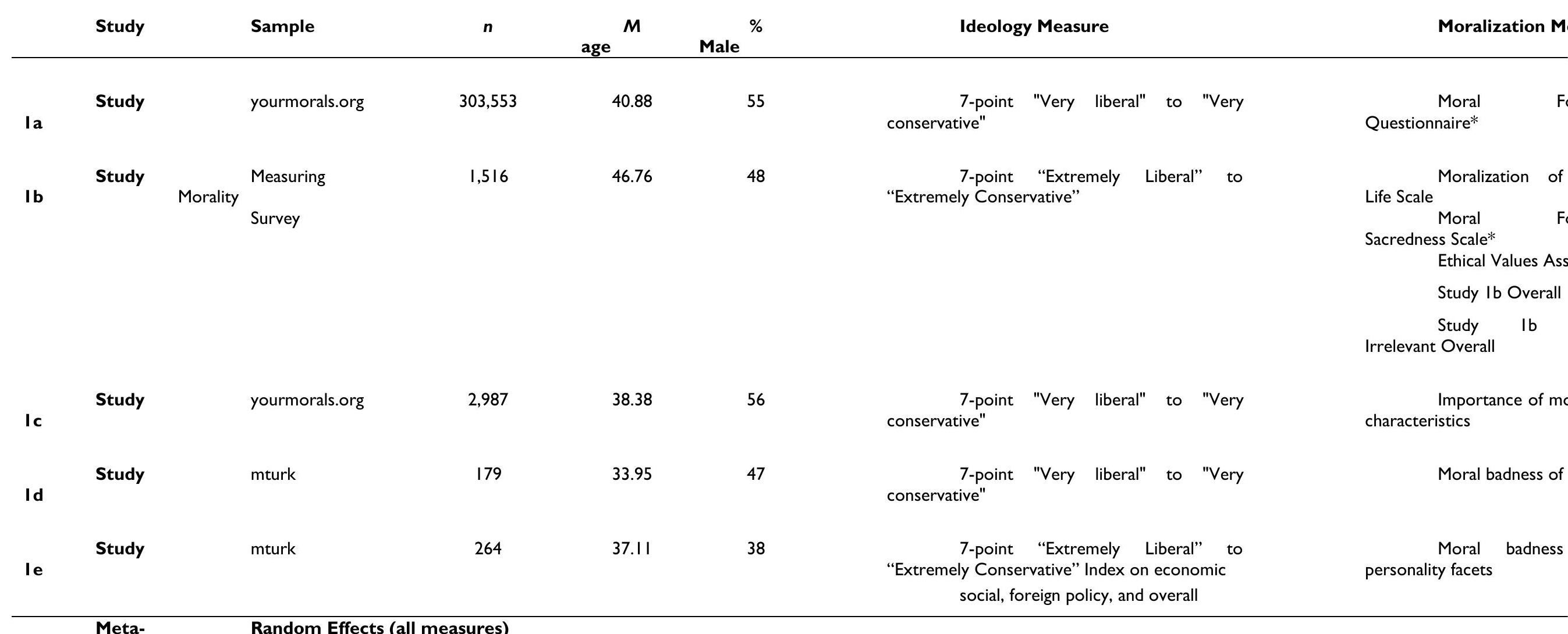




\section{Discussion}

This meta-analysis adds to the body of research demonstrating that conservatives have stronger tendencies to moralize than liberals. This was true overall, and crucially, was true even for moralization measures containing zero political content (e.g., moral badness ratings of faces and personality traits). Thus, these results support a premise of our present hypothesis and justifies our examination of whether conservative moralization may explain —in part—conservatives' stronger beliefs in free will and personal responsibility.

\section{Study 2}

Study 2 employed individual difference measures to examine the relationships among political ideology, free will belief, and moralization. We conducted a preliminary test of our hypothesis by assessing whether tendencies to hold others morally responsible for blameworthy actions mediate the relationship between conservative ideology and belief in free will. The Free Will and Determinism Scale-Plus (FAD-Plus; Paulhus \& Carey, 20II) is probably the most widely used free will belief scale, but it is routinely criticized for confounding free will and moral responsibility for blameworthy actions (Clark et al., 20I4; Clark, Winegard, \& Baumeister, 2019; Monroe \&Ysidron, 2019). Items such as "Criminals are totally responsible for the bad things they do" and "People must take full responsibility for any bad choices they make" measure beliefs about how blameworthy people generally are for bad actions more than beliefs about freedom and control. Other items, however, exhibit higher prima facie validity, such as "People have complete control over the decisions they make" and "Strength of mind can always overcome the body's desires." We did not have access to large scale data with the modern FAD-Plus, but we did have access to large scale data with the original FAD (Paulhus \& Margesson, 1994), which is identical on five of the seven items and similarly contains four face valid items and three general blame items. We decided to leverage the flaws of this scale to examine whether tendencies to blame (using the blameworthy scale items) statistically mediate the relationship between conservative political ideology and free will belief (using the face valid items). 2 There are two limitations to this approach. First, the FAD items were likely selected by scale developers to intercorrelate and this weakens their appropriateness for mediation analysis. Second, causal orders cannot be inferred from mediation analyses of this sort (see our later studies, which experimentally test whether manipulating moral blame motives influence free will judgments, for such causal evidence). Nevertheless, this analysis is at least suggestive that a proportion of the relationship between more conservative political ideology and free will beliefs can be linked to general blame tendencies.

\section{Method}

Participants $\left(n=14,708 ; 38 \%\right.$ Female; $M_{\text {age }}=34.80, S D=15.88 ; 73 \%$ from the U.S., the remaining from more than 100 countries) were recruited through yourmorals.org. This study received ethical approval from the IRB of UC Irvine ("Moral Psychology on the Internet"; Protocol \#2007-5740). Demographic information is collected at registration including age, sex, education, religious attendance, and political orientation. After registration, visitors self-select 
to take one or more surveys from a list of over 50. For inclusion, participants had to have completed the free will subscale from the Free Will and Determinism scale (FAD; Paulhus \& Margesson, 1994), which was broken down into one free will belief subscale $(\alpha=.69)$ containing four items (e.g., "People have complete control over the decisions they make.") and one blame subscale $(\alpha=.7 I)$ containing three items (e.g., "Criminals are totally responsible for the bad things they do."), each rated on a 5-point scale from "strongly disagree" to "strongly agree". All participants who completed the FAD were included if they also reported their political ideology on a 7-point scale from "Extremely Liberal" to "Extremely Conservative." The sample leaned liberal $(M=2.99, S D=1.69)$, with $66.4 \%$ below the moderate midpoint, $13.8 \%$ at the moderate midpoint, and $19.7 \%$ above the moderate midpoint.

\section{Results}

More conservative ideology was positively correlated with free will belief, $r=.35 \mathrm{I}, p$ $<.00 \mathrm{I}$, and blame tendencies, $r=.427, p<.00 \mathrm{I}$, and free will belief and blame tendencies were positively correlated, $r=.527, p<.00 \mathrm{I}$. We conducted two bootstrap mediation analyses ( 10,000 resamples) testing whether blame tendencies statistically mediated the relationship between ideology and free will beliefs both with and without demographic controls (age, sex, education, and religious attendance). Both mediations were highly significant, without controls (see Figure I), $b=.100$, se $=.002,95 \% \mathrm{Cl}[.095-.104], Z=41.52, p<.000 \mathrm{I}$, and with all controls, $b=.097, \mathrm{se}=.003,95 \% \mathrm{Cl}[.090-.103], Z=29.15, p<.000 \mathrm{I}$ (being younger, female, less educated, and more religious all significantly predicted stronger free will belief; being older, female, and less educated significantly predicted stronger blame tendencies [religiosity did not significantly predict blame tendencies]).

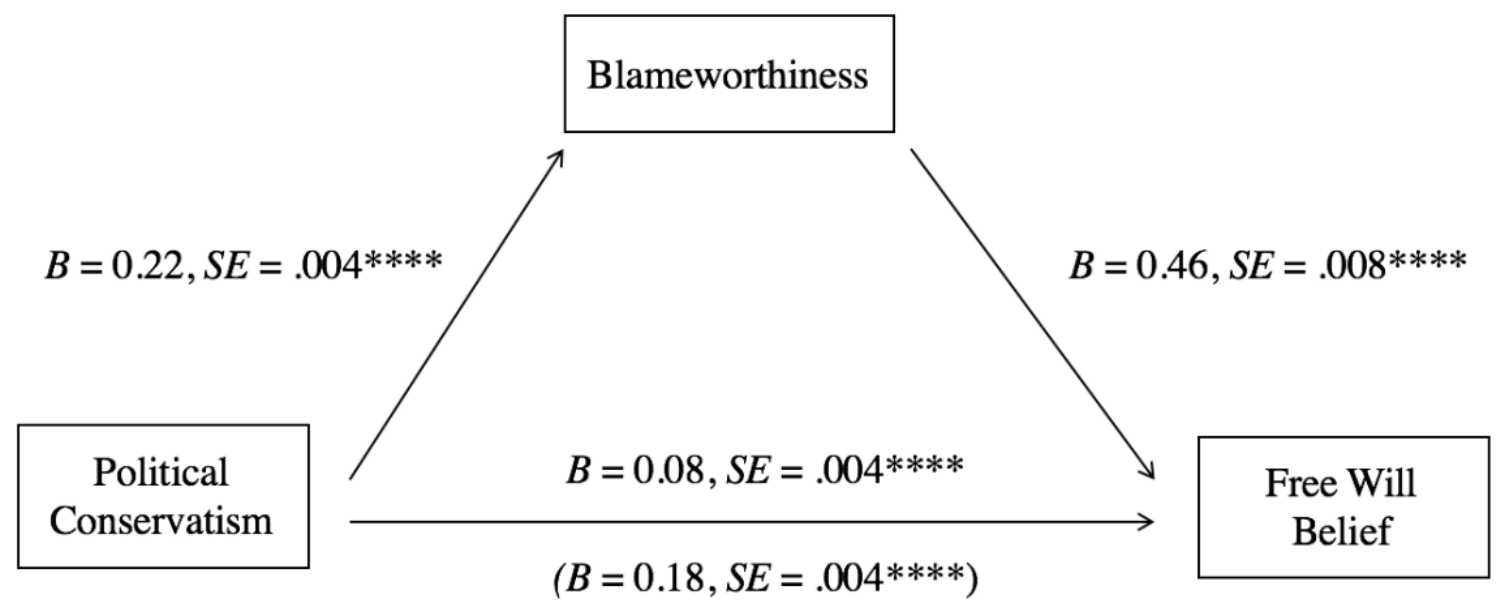

Figure I. Indirect effect of more conservative political ideology on free will belief through blame in Study 2. Note. ${ }^{* * *}$ indicates $p<.001$.

\section{Discussion}

Study 2 provided individual difference evidence consistent with our hypothesis. Stronger beliefs that people are morally responsible for their bad behaviors statistically mediated the relationship between more conservative ideology and stronger beliefs in free will-both with and without relevant demographic controls. This suggests that higher tendencies to blame account for a proportion of the relationship between more conservative 
political ideology and free will beliefs. Of course, correlational studies cannot supply evidence of causal relationships and so our later studies manipulate blame desires directly to test how these differentially impact the free will judgments of conservatives and liberals.

\section{Study 3}

In Study I we have demonstrated that conservatives moralize a much broader range of actions than liberals, and in Study 2 that conservatives report higher general belief in free will, and that this association of ideology with free will belief is partially mediated by beliefs that others are morally responsible for their bad actions. In Study 3 we wanted to turn away from reports of general, abstract belief in free will to look at attributions of free will for specific events, using the opportunity to look at attributions of free will for both positive and negative events.

To our knowledge, no research has been conducted on a comparison of political differences in attributions of free will for positive events. This is surprising, though, because the comparison between attributions for positive and negative events sets up an interesting test of two alternative explanations for what may be driving the existing liberal-conservative differences in endorsement of free will. If conservatives are dispositionally inclined to hold a stronger belief that people have free will than liberals, then conservatives should endorse and attribute greater free will regardless of whether their actions lead to good or bad outcomes. However, if differences in free will belief are in part motivated by moral judgments of wrongness and a desire to blame, then we should see an interaction between political affiliation and the positivity or negativity of the event. Since conservatives have stronger tendencies to moralize, they should show a stronger negativity bias in their free will attributions - that is, attributing more free will to actions with negative rather than positive outcomes - than would liberals. In Study 3, we test this.

\section{Method}

Open Science and Ethics Statement. Our design, hypotheses, and analysis plan were all pre-registered at the Open Science Framework. For this study and all subsequent ones, we report all measures, manipulations, and exclusions, and results, analysis code, and experiment materials are available for download at: https://osf.io/ny $82 \mathrm{c} /$. This study received ethical approval from the IRB of the University of British Columbia ("Social Impacts of Emerging Technology"; Protocol \#H 18-02727).

Participants. We originally recruited I 46 American participants online using MTurk. On the recommendation of an anonymous reviewer and editor, we subsequently conducted a pre-registered second wave of data collection to maximise power.We report in the main text results using the original sample combined with the new one, which gave us a final sample of 444 participants ( 88 female, $M_{\text {age }}=35$ ). On average, participants were slightly left-of-center on a I-7 scale $(M=3.89, S D=1.65)$, with I 88 Democrats and I 3 I Republicans (the remaining were neither).

Design. We had a fully within-subjects design, where participants were asked to rate how much free will someone had for six distinct situations. Three of these were negative (the material living conditions of the homeless, drug addicts' addictions, a man imprisoned for participating in gang violence) and three were positive (the financial success of investment 
bankers, students' admission to elite universities, a famous musician winning a Grammy award). These items were selected simply because they appeared to be unambiguously positive or negative, but later studies will more systematically pretest the selected target actions. For each item, participants rated how much $(\mathrm{a})$ responsibility $(I=$ Not at all personally responsible to $7=$ Completely personally responsible);3 (b) control ( $I$ = Not at all in control; 7 = Completely in control); and (c) free will ( $I=$ No free will at all; $7=$ Complete free will) the different groups or individuals concerned had for their current situation. These scores were aggregated to form a reliable overall measure of free will attributions for both positive $(\alpha=.83)$ and negative situations $(\alpha$ $=.82)$.

Analysis. We collected and analyzed one wave of data prior to our initial submission for publication. At the request of a reviewer and our editor, we then conducted another wave of data collection before completing the final analyses. To account for this sequential testing and ensure full transparency, we have taken three approaches. First, we have simply reported the results in full for the original sample in the supplemental results at the OSF. Second, we have employed the technique of adjusting our significance levels for sequential testing. Following the guide provided by Lakens (2014), we have used a linear alpha spending technique to obtain a revised significance level of $p \leq .038$ that accounts for the sequential testing. While an increasingly common technique in social psychology that easily allows the reader to see whether our key result is significant at this revised level, the strategy is formally inappropriate - though still acceptable - because we did not pre-specify that we would conduct two waves of data collection and analysis. Given this, the third strategy we have used is to compute a paugmented statistic (Sagarin et al., 2014), a technique explicitly designed for cases where the sample size has been increased post-hoc after initial data analysis. This statistic consists of a range of values greater than $p \leq .05$ and represents the magnitude of the resulting Type I error inflation as a result of our increased data collection.

\section{Results}

We used regression to look at whether political orientation (continuous betweensubjects) predicted free will attributions, and whether this differed for the positive and negative events (as a within-subjects variable, $0=$ positive, $I=$ negative). In this and all subsequent studies, we report semi-partial $r$ (the proportion of the variance in free will attributions uniquely explained by the indicated predictor) as estimates of effect sizes (except for the mixed withinbetween interaction here, for which the semi partial $r$ is not available).

Results using the full sample revealed a significant interaction between valence condition and political ideology, $b=-.03, \mathrm{SE}=.0 \mathrm{I}, t=-2.16, p=.03 \mathrm{I}, 95 \% \mathrm{Cl}[-.06, .00]$, Pseudo$R^{2}=0.49$, paugmented $=[.053, .073]$. Though a small effect, as can be seen in see Figure 2 , this interaction was significant even at our lower threshold of significance, $p<.038$, accounting for the sequential data collection (Lakens, 20I4). This interaction supplemented a main effect of

\footnotetext{
3 A reviewer questioned the appropriateness of a responsibility question in our index of free will attributions. Here, we are precisely interested in motivated increases in free will attributions for purposes of increased personal responsibility, so it seemed appropriate for the question at hand. But we also cross-checked our main results removing this responsibility item, and this did not impact the statistical significance of any of our main results. Thus, even removing this item, the interpretation of our data remains unchanged.
} 
ideology whereby conservative ideology was associated with greater free will attributions overall, $b=.26, S E=.02, t=12.06, p<.00 \mathrm{I}, 95 \% \mathrm{Cl}[.22, .3 \mathrm{I}]$, and no overall difference in attributions of free will for the positive or negative events, $b=-.02, S E=.03, t=0.58, p=.56$, $95 \% \mathrm{Cl}[-.04, .07]$. Specifically, the relationship between conservative ideology and attributions of free will was stronger for negative events, $b=.30, S E=.03, t=11.24, p<.001,95 \% \mathrm{Cl}$ $[.24, .35], R_{2}=0.22$, semi-partial $r=0.47$, than for positive events, $b=.23, S E=.03, t=8.78, p$ $<.001,95 \% \mathrm{Cl}[.18, .28], \mathrm{R}_{2}=0.15$, semi-partial $r=0.39$ (see Figure 2).

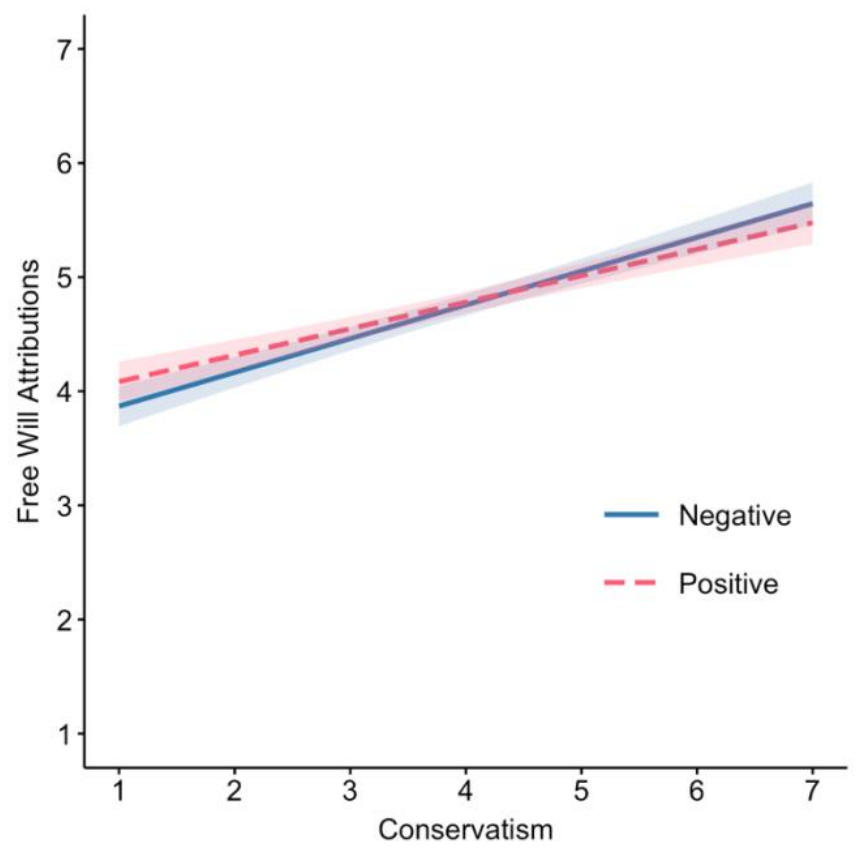

Figure 2. Attributions of free will as a function of participant political ideology, for positive and negative events separately. (Study 3 ).

\section{Discussion}

In Study 3 we looked at whether political ideology was associated with attributions of free will for specific events. We found that political ideology indeed predicted higher free will attributions overall, and this relationship appeared stronger for negative situations than positive ones (though, contra our pre-registered hypothesis, the political difference was only reduced - rather than absent entirely-among positive situations). Conceptually, the capacity for free will should hold whether one experiences a good or bad outcome, and so if ideology is genuinely related to an abstract belief in free will, there should have been no difference depending on the valence of the outcomes. That we observed a small-but-significant interaction whereby conservatism predicted higher attributions of free will to a stronger degree for the negative than the positive events, however, suggests that free will attributions are not merely reflecting some dispositional variance in a belief in human autonomy, but instead a more basic, social psychological phenomenon - likely one relating to blame.

One important limitation of this study, though, is that we did not control for perceptions of wrongness of the events. We assume that Republicans attributed more free will to the negative events - being homeless, being a drug addict, and being imprisoned for gang violence-because these are all situations that conservatives tend to find more morally 
objectionable than do liberals (e.g., Graham et al., 2009). If free will judgments only differ by political ideology when there are differences in perceived moral wrongness, then these differences should be eliminated when the perceived wrongness of the event is matched for both conservatives and liberals.

\section{Study 4}

Study 4 sought to further chart the boundaries of the association between free will and political ideology by looking at attributions of free will for specific events that were pretested to be equally immoral for liberals and conservatives. If political differences in free will belief are mainly derived from differences in moralizing, these free will differences should be eliminated when looking at items judged as equally wrong by both liberals and conservatives. On the other hand, if political ideology is more abstractly and generally related to free will belief, then conservatism should be associated with higher free will attributions across different events, even those that are judged as equally wrong by both liberals and conservatives.

While most prior work has focused on attributions for morally negative events only (see Study 3), we wanted to look at free will attributions for a range of both moral and nonmoral, and positive and negative events to test the generality of our hypothesis. If our hypothesis that political ideology is related to free will belief because of differences in the scope of the moral domain is correct, we should observe roughly equivalent attributions of free will between liberals and conservatives for any type of action that has roughly equal moral significance for liberals and conservatives.

\section{Method}

Pre-Testing. We pretested items for use in Study 4 that did not significantly differ in perceived morality (or valence) based on political orientation. To do this, we recruited 109 MTurk participants (34 female, $M_{\text {age }}=36$ ), though five participants were excluded for failing two attention checks (e.g., "Please click Scale Point I to confirm you're paying attention"), leaving 104 participants in the analysis. On a I-7 scale (7 indicating stronger conservatism), participants were on average slightly left-of-center $(M=3.53)$, with slightly liberal views on social issues $(M=3.19)$ and moderate views on economic issues $(M=3.88)$. All main political positions were represented, with 21 Republicans, 39 Democrats, and 43 Independents.

Participants were required to rate a series of $5 \mathrm{I}$ events and occurrences, I) for how positive or negative it was $(-100=$ negative; $0=$ neutral; $100=$ positive); and 2$)$ for how moral it was: is the action morally bad, morally good, or irrelevant to morality? $(-100=$ morally bad; $0=$ morally irrelevant; $100=$ morally positive). All of these items were devised by the researchers to be as politically neutral as possible, including a range of both positive and negative items, and moral and non-moral items. The list of all items, along with mean scores and correlations with ideology, can be seen on the OSF. To select the final items to use for the main study we used a statistical cut-off point $(r<.10 ; p>.40)$ to ensure conservatives and liberals did not rate the item as differentially positive or negative, or differentially morally relevant or not. Based on this, we selected 20 items: five that were moral and positive; five that were moral and negative; five that were non-moral and positive; and five that were non-moral and negative.

Open Science and Ethics Statement. For the main study, we report all measures, manipulations, and exclusions. Results, analysis code, and experiment materials are available 
for download at the OSF. This study received ethical approval from the IRB of the University of Oregon ("The Effect of Culture on Attitudes and Outcomes"; Protocol \#101620 I2.023).

Participants. For the main study, 647 American participants completed the survey online using MTurk. 18 participants were excluded from analysis for taking the survey more than once, leaving a final sample of 629 participants (269 female; $M_{\text {age }}=35$ ). Our sample size was determined by available funds and a goal to recruit at least I50 participants per each of the four conditions.

Design. Our experimental design had a 2 (morality: morally relevant vs. non-moral) $x$ 2 (valence: positive vs. negative) experimental design, where in each condition participants were given the five events obtained from pre-testing and asked to indicate how much free will they perceived the actor to have. Some participants read events that were - equally to liberal and conservatives - seen as positive and morally-relevant (e.g., "Working one day a week at a soup kitchen"); others read events that were negative and morally-relevant (e.g., "Spreading malicious rumours about a co-worker"); others read events that were positive but non-moral (e.g., "Making money from a smart investment"); and the remaining participants read events that were negative but non-moral (e.g., "Failing a college exam"). Full items can be seen on the OSF. The order of the events and the five dependent variables for each event were all randomized for each participant to avoid potential order effects.

Measures. For each of the five events, participants rated the degree of free will that they perceived the actor to have using five items: "How much control would someone have over __"; How much responsibility would someone have for __"; "To what extent would someone who did _ have exercised free will?"; "To what extent is someone who performing an action that is freely chosen?"; "To what extent would someone who did _ have been able to have made other choices and not done this?" rated on 7-point scales from "not at all" to "very much." Within each condition these five items showed high internal consistency ( $\alpha$ 's > .88) and so were aggregated together to form an overall measure of free will attributions (i.e., all 5 DVs for all 5 items).

Participants indicated their political ideology on two scales: one measuring social conservatism, and one measuring economic conservatism, rated on 7-point scales from "very liberal" to "very conservative," which were combined into an index of overall political ideology $(\alpha=.73)$. Finally, participants completed the free will subscale of the FAD+.

\section{Results}

First, we looked at the potential interactive effect of political orientation with attributions of free will for events that were either positive or negative, and either morally relevant or non-moral. We used a regression-based procedure to examine the effects of morality $(-\mathrm{I}=$ non-moral; $\mathrm{I}$ = morally relevant $)$, valence $(-\mathrm{I}=$ positive, $\mathrm{I}$ = negative $)$, and participant political orientation (centered) on attributions of free will. As expected, there was no main effect of political ideology on ascriptions of free will, $b=.00, S E=.02, t=-0.09, p=$ $0.93,95 \% \mathrm{Cl}[-.04, .03]$, semi-partial $r=-.00$. Moreover, there was no three-way interaction of morality, valence, and political orientation, $b=-.03, S E=.02, t=-1.63, p=0.10,95 \% \mathrm{Cl}[-.07, .0 \mathrm{I}]$, $R_{2}=0.28$, semi-partial $r=-.06$, and no interaction of political orientation with whether the event was moral or non-moral, $b=-.03, \mathrm{SE}=.02, t=-\mathrm{I} .5 \mathrm{I}, p=0.13,95 \% \mathrm{Cl}[-.06, .0 \mathrm{I}]$, semipartial $r=-.05$, or of political orientation with whether the event was positive or negative, $b$ 
$=.01, S E=.02, t=0.59, p=0.55,95 \% \mathrm{Cl}[-.02, .05]$, semi-partial $r=.02$. That is, for these items that we had pre-tested to be equally matched in morality (and valence) for liberals and conservatives, there was no effect of political ideology on specific free will attributions. Indeed, for the 10 items pre-tested to be equally morally relevant to liberals and conservatives, there was not a single significant correlation between political orientation and free will attributions. This was despite replicating our finding from Study 2 (and previous work by Carey and Paulhus, 20I3), that conservative political ideology was significantly positively correlated with scores on the free will subscale of the FAD $+(r=0.29, p<.00 \mathrm{I})$, with conservatives showing higher endorsement of both the more abstract $(r=0.24, p<.00 \mathrm{I})$ and moral responsibility focused $(r=0.28, p<.001)$ items of the scale. Scores on the FAD+ were significantly correlated with free will attributions across the events $(r=0.39, p<.00 \mathrm{I})$.

In other words, even though conservatives consistently report stronger beliefs in free will than liberals, and stronger beliefs in free will are associated with stronger attributions of free will in general, conservatives do not attribute more free will than liberals to actions that they perceive as equally as morally wrong as liberals. Thus, when the moral relevance of actions is controlled for, political differences in free will attributions are eliminated.

\section{Discussion}

In Study 4 we explored whether we might be able to "break" the association of political ideology and free will by looking at actions judged to be morally equivalent between liberals and conservatives. If political ideology is related to free will belief primarily because of differences in the scope of the moral domain, we reasoned, these differences should be markedly reduced when looking at items judged as equally wrong by both liberals and conservatives. Indeed, we found the differences were eliminated altogether: though we again replicated that conservative ideology was significantly positively associated with an abstract agreement that humans have free will, there were no differences in specific attributions of free will for actions that were pre-tested to be equally morally valenced for liberals and conservatives. These results are consistent with our hypothesis that differences in conservatives' and liberals' perceptions of free will may be partially due to differences in moralization, rather than representing any generalized, abstract belief that human behaviors are freely chosen.

One potential concern with Study 4, however, is that we predicted (and found) a null effect. Our findings are consistent with the idea that differences in conservatives' and liberals' perceptions of free will are partially due to differences in moralization, and inconsistent with the idea that conservatives attribute more free will regardless of the moral content. Nonetheless, because it is impossible to prove a null hypothesis by rejecting it, we cannot make firm conclusions about the motivated basis of free will on the basis of such a null finding. In Study 5, then, we aimed to conduct a stronger test of our hypothesis by looking at whether the association of conservatism with greater free will could be reversed, not just eliminated.

\section{Study 5}


If the typical tendency for conservatives to endorse stronger beliefs in free will is due to a motivation to blame, rather than a stable personality characteristic, this tendency ought to reverse when confronted with behaviors that conservatives perceive as less morally wrong than their liberal counterparts. We tested this prediction in Study 5.

\section{Method}

Pre-Testing. We first pretested for items that political conservatives would see as less morally wrong than liberals. American MTurk participants $\left(n=100 ; 44\right.$ female, $M_{\text {age }}=32$ ) rated how liberal or conservative they were on a $1-7$ scale, with participants being slightly liberal on average $(M=3.44)$, with slightly more liberal views on social issues $(M=3.07)$ and more moderate views on economic issues $(M=3.80)$. All main political parties were represented, with 13 Republicans, 47 Democrats, and 36 Independents. To help select our items for the main study, participants rated the moral wrongness of a series of 25 events and behaviors that were devised by the research team to specifically cover things that liberals typically are more morally concerned about than conservatives (e.g., animal welfare, recycling, prejudiced behavior [Graham et al. 2009]). The list of all items, along with mean scores and correlations with ideology, can be seen on the OSF.

From participants' ratings, we selected five items based on both the strength of correlation between political ideology and wrongness (all $p s<.05$; all $r s>.22$ ), and the mean wrongness (to avoid ceiling or floor effects: Ms between 3.62 and 4.95 on a I-7 scale). It is illuminating to note that, for 10 out of the 25 items, there were no significant correlations of political orientation with ratings of wrongness. In other words, even when we intentionally created items to capture things that liberals, but not conservatives, are typically outraged by, conservatives judged $40 \%$ of the items as equally wrong as liberals did.

Open Science and Ethics Statement. For the main study, we report all measures, manipulations, and exclusions. Results, analysis code, and experiment materials are available for download from the OSF. This study received ethical approval from the IRB of the University of Oregon ("The Effect of Culture on Attitudes and Outcomes"; Protocol \#10162012.023).

Participants. For the main study, $5 \mathrm{I} 3$ American participants completed the survey online via MTurk.4 Participants were excluded from data analysis if they took the survey more than once $(n=5)$ or failed one or both of two simple checks in which they had to select a certain scale-point in the FAD+, and then indicate at the end of the survey which event they did not answer questions about $(n=36)$. This left a final sample of 472 participants ( 237 female; $M_{\text {age }}=37$ years). As in Study 3, we used the same combination of sequential testing (Lakens, 2014) and a paugmented statistic (Sagarin et al., 2014) to account for the two stages of data collection and analysis.

\footnotetext{
4 We originally recruited 204 participants and analyzed the results (after exclusions, $n=193$ ), and our results were not statistically significant. To explore whether this outcome was due to any problems with the specific items, we decided to run another pilot study. However, when attempting to conduct this pilot, a technical error resulted in recruitment of 275 more participants for the present study instead of the new pilot. We then reanalyzed the data with the updated sample and found statistically significant results supporting our hypotheses, suggesting that the non-significant effects in the initial analysis were explained by a lack of power. In the interest of full transparency, we report both sets of results in full at the OSF, and then use the same combination of sequential testing and the paugmented statistic to account for our waves of data collection.
} 
Design. In the main study, participants were given a list of five events that pre-testing revealed would be perceived as more wrong by liberals: I) "Robert sends a formal complaint to his child's school after finding that his child's kindergarten teacher is transgendered"; 2) "Sarah uses make-up products that are tested on animals"; 3) "In conversation with a fellow student, John finds out that the student is gay. John immediately tells the student that he will pray for him"; 4) "Garrett manages an upscale members club where only young and attractive women are employed"; and 5) "Riley is remotely piloting military drones above Afghanistan. She has been ordered to target a compound believed to hold terrorists, but she knows there may also be civilians. A few hours after she pilots her drone to attack the compound, she finds out that 8 suspected terrorists and 5 children died in the resulting explosion".

Each of the five events were presented in randomized order. Participants rated how much free will, responsibility, control, and free choice the actor had, and these four items were aggregated to form a measure of free will attributions, as for Study $3(\alpha=.85)$. Participants also rated how wrong they perceived the described action to be on a I-7 scale from "not at all wrong" to "very wrong." Last, participants again completed the free will subscale of the FAD+ and reported their political ideology on the same two 7-point economic and social conservatism used in previous studies.

\section{Results}

Supporting our hypothesis, for actions in which political liberals were more motivated to assign blame (correlation of rated wrongness with ideology: $r=-.47, p<.00 \mathrm{I}$ ), conservative political ideology (mean-centred) negatively predicted free will, $b=-.05, S E=.02, t=-2.73, p$ $=.007,95 \% \mathrm{Cl}[-.09,-.02], \mathrm{R}_{2}=0.02$, semi-partial $r=-.12$, paugmented $=[.053, .054]$. This interaction was significant even at our lower threshold of significance, $p<.036$, accounting for the sequential data collection (Lakens, 20I4). That is, for these actions that political liberals saw as more wrong, it was political liberalism that predicted greater free will attributions. This was despite political conservativism again being significantly positively correlated with scores on the free will subscale of the FAD+ $(r=0.19, p<.001)$, with conservatives showing higher endorsement of both the more abstract $(r=0.16, p<.001)$ and moral responsibility focused $(r=0.19, p<.001)$ items of the scale. That is, whereas political conservatives again reported higher general, abstract belief in free will, when it came to attributing free will for specific events that conservatives found less morally wrong than liberals, conservatives attributed less free will.

Supporting the idea that differences in moralization underpin the specific free will attributions, we found that when adding perceived moral wrongness (mean-centred) to the model, political ideology no longer predicted ascriptions of free will, $b=-.03, S E=.02, t=$ I.I8, $p=.24,95 \% \mathrm{Cl}[-.07, .02]$, semi-partial $r=.05$, with only reported moral wrongness significantly predicting free will attributions, $b=.08, S E=.03, t=2.6 \mathrm{I}, p=.009,95 \% \mathrm{Cl}[.02, .13]$, semi-partial $r=.12$ - thus implicating wrongness as the factor driving the relationship between free will and political ideology, at least in part. Finally, we conducted a mediation analysis (10,000 resamples) which revealed that wrongness judgments significantly statistically mediated the relationship between political ideology and attributions of free will, $b=-.03$, se $=.0 \mathrm{I}, 95 \% \mathrm{Cl}[-.05,-0.0 \mathrm{I}], Z=-2.55, p=.0 \mathrm{II}$.

\section{Discussion}


The results of Studies 4 and 5 suggest that the conservative tendency to ascribe more free will than liberals does not (just) reflect a stable personality trait, but rather corresponds to the perceived moral wrongness of actions. While conservatives consistently report higher general, abstract belief in free will as measured by the FAD+, a different pattern emerges when it comes to judgments of free will for specific events. For events that liberals and conservatives were shown to see as equally morally wrong or praiseworthy, conservatives and liberals attributed the same degree of free will (Study 4), and for events that liberals see as more morally wrong than conservatives, it was liberals that attributed more free will (Study 5).

For Study 5, we wish to emphasize that these results only reached statistical significance when using a larger sample than originally intended, indicating a smaller effect size for the relationship between perceived moral wrongness and free will attributions for political liberals than for political conservatives. Although we had not predicted this outcome in advance, it seems consistent with our theoretical framework that liberals' motivated free will judgments would be weaker than conservatives' due to their relatively weaker tendency to perceive actions as morally significant. While it would be surprising if liberals did not exhibit any motivated social cognition whatsoever (i.e., if they did not attribute more free will to actions they perceived as wrong; Ditto et al., 2019a, 2019b), it would be equally surprising if this tendency were equally as strong as it is for conservatives given the generally weaker tendencies to moralize among liberals. A tempered or weaker effect, then, might be expected for liberals.

\section{Study 6}

Thus far we have shown that conservatives moralize a wide variety of events more than liberals (Study I), and while they generally report greater free will beliefs than do liberals (Studies 2-5), this tendency may be in part motivated by a greater desire to blame since conservatives show no difference in free will attributions for actions seen as equally wrong as liberals there is no difference in free will attributions (Study 4), and show lower free will attributions for actions they find less wrong than liberals (Study 5). What we have not shown yet, though, is whether a) conservatives would attribute more free will for events that they found more morally wrong than liberals; and b) whether judgments of moral wrongness for specific actions would mediate the relationship between conservative political orientation and free will attributions.

In Study 6, therefore, we built upon the body of evidence from Studies 2-5 to look at attributions of free will both for events that conservatives find more wrong, and for politically neutral events in which there is no difference between conservatives and liberals' perceptions of wrongness. Our prediction was that conservatives would only attribute more free will for the events they found morally wrong, and that this relationship between conservative ideology and free will attributions would be statistically mediated by how wrong participants judged the behavior to be. We test this in two separate studies: an initial test (Study 6a), and then a later, pre-registered replication with a larger sample size (Study 6b).

\section{Method}

Pre-Testing. As in the previous studies, we pretested items for use in Study 6. Here, however, we sought to identify items that either did or did not differ in perceived wrongness 
as a function of political orientation. American participants on MTurk ( $n=110 ; 44$ female, Mage = 34) rated how liberal or conservative they were on a 1-7 scale. Participants were again slightly liberal on average $(M=3.30)$, with more liberal views on social issues $(M=2.95)$ and slightly more moderate views on economic issues $(M=3.65)$. All main political parties were represented, with 22 Republicans, 42 Democrats, and 40 Independents. Participants were then required to rate a series of 38 events and occurrences for how morally wrong they perceived them to be.

In order to obtain politically neutral events, most of the items were drawn from the 30-item Moralization of Everyday Life Scale (MELS; Lovett et al., 20I2). This consists of 30 common and everyday moral violations, the vast majority of which lack any clear connection to political ideology (e.g., "Ava parks in a handicapped zone even though she is not handicapped" or "Joseph starts smoking a cigarette in a non-smoking section of a restaurant"). We primarily drew from the MELS to obtain the politically neutral items in order to reduce any unconscious bias that could exist when creating items. In order to obtain items that conservatives would judge as more wrong, we included 8 additional items that we devised ourselves to parallel the format of the MELS items, but with content that we assumed would be judged differently by conservatives and liberals (e.g., "Doug pretends to be ill to avoid being sent to war").

To select the five 'politically neutral' items on which conservatives and liberals did not differ in judgments of wrongness, we used the same statistical cut-off point as in Study 4 ( $r$ $<.10 ; p>.40$ ). To select the five 'conservative wrong' items, we used a statistical cut-off point $(r>.40 ; p<.005)$ to ensure that items were significantly associated with political orientation such that that conservatives judged the items as significantly more wrong. Based on these criteria, we selected 5 items from each category to use as the 'Conservative Wrong' dependent measures in this study (see Table 2).

The list of full items and correlations with political ideology can be seen in at the OSF. Note that the items used in both Study $6 \mathrm{a}$ and $6 \mathrm{~b}$ were the same, but we changed some of the names of the protagonists in Study 6b. In the time between conducting our initial study and the replication, a new paper was published that looked at the effect of names in vignette experiments, providing a list of names matched in perceived age, warmth, and competence (Newman et al., 2018). To eliminate any unintentional influence of the names we had chosen, in Study $6 \mathrm{~b}$ we therefore used names from this list.

Table 2: Items by Condition for Study 6b

Conservative Wrong Politically Neutral

Jennifer has her second abortion in two years
Evelyn is taking a casual walk around the block on a snowy day, and she notices a driver whose car is stuck in the snow. She keeps walking rather than stopping to see if she can help. 
David becomes addicted to drugs and drops out of college.

Ann is out to dinner with some friends and has some gas pains in her stomach. She decides to release gas, even though she knows it will make an awful smell.

Sarah made an abstinence-untilmarriage vow when she was 16, but now she's 18 and in college, and she has sex with a boyfriend with whom she's in love. John pretends to be ill to avoid being sent to war

\section{Method}

\section{Open Science}

We report all measures, manipulations, and exclusions. Results, analysis code, and experiment materials are available for download from the OSF. This study received ethical approval from the IRB of the University of Oregon ("The Effect of Culture on Attitudes and Outcomes"; Protocol \#I0162012.023).

Participants. 30I American participants completed the survey online via MTurk. Participants were excluded from data analysis if they took the survey more than once $(n=1)$ or failed one or both of two simple checks in which they had to select a certain scale-point in the FAD+, and then indicate at the end of the survey which event they did not answer questions about $(n=4)$. This left a final sample of 294 participants ( 121 female; $M_{\text {age }}=32$ years). Sample size was determined by available funds and a goal of approximately 150 participants per condition.

Design. Participants were randomly assigned to one of two conditions (Conservative Wrong; Politically Neutral) in a between-subjects design. Participants were given a list of five events that pre-testing showed were either differentially perceived to be more morally wrong to conservatives than to liberals (Conservative Wrong), or five items on which conservatives and liberals did not differ in their perceptions of wrongness (Politically Neutral). For each of these five items (see Table 2), participants were asked to rate the degree of wrongness and free will the actor had for each event using the same measures as Study 5 (how much 
responsibility, control, free will, and ability to do otherwise). The order of the events and the five dependent variables for each event were all randomized, and at the end participants completed the free will subscale of the FAD+.

\section{Results}

First, we again looked at correlations of political conservativism with general free will belief. As in the previous studies, conservatism was significantly positively correlated with scores on the free will subscale of the FAD $+(r=.38, p<.001)$, with conservatives showing higher endorsement of both the more abstract $(r=.33, p<.001)$ and moral responsibility focused $(r=.37 p<.001)$ items of the scale.

Next, we checked whether political orientation was indeed associated with greater judgments of moral wrongness in the 'conservative wrong' condition. Despite pre-testing the political neutral events to be equally morally wrong for liberals and conservatives, we actually found in the main study that conservatives rated the 'politically neutral' events to be more wrong ( $r=.24, p=.003)$, though unsurprisingly this relationship was much weaker than in the 'conservative wrong' condition $(r=.57, p<.00 \mathrm{I})$. Across participants, wrongness ratings were positively associated with free will attributions for the conservative wrong events $(r=.23, p$ $=.005)$, but not the politically neutral events $(r=.02, p=.77)$.

We then turned to our key analyses and examined how morality condition $(-\mathrm{I}=$ politically neutral; I = conservative wrong) and participant political orientation (centered) interacted to predict attributions of free will. We observed a significant interaction of political ideology and morality condition, $b=.05, \mathrm{SE}=.02, t=2.18, p=.030,95 \% \mathrm{Cl}[.00, .09], \mathrm{R}_{2}=0.2 \mathrm{I}$, semi-partial $r=0.11$, whereby as predicted conservatives attributed more free will for the 'conservative wrong' items, $b=.08, \mathrm{SE}=.03, t=2.33, p=.021,95 \% \mathrm{Cl}[.0 \mathrm{I}, .15], \mathrm{R}_{2}=.04$, semipartial $r=.19$, but there was no difference in attributions of free will for the politically neutral events, $b=-.02, \mathrm{SE}=.03, t=0.57, p=.57,95 \% \mathrm{Cl}[-.07, .04], \mathrm{R}_{2}=.00$, semi-partial $r=-.05$.

Finally, we tested our prediction that wrongness judgments would statistically mediate the effect of political orientation on attributions of free will in the 'conservative wrong' condition. We began by conducting a moderated mediation analysis looking at whether the wrongness condition moderated the mediation of wrongness judgments on the path from political orientation to attributions of free will. We observed significant moderation, $95 \% \mathrm{Cl}$ $[.03, .10]$, which we then probed by looking at the mediation of wrongness judgments within each condition (10,000 resamples). We found that the mediation effect was stronger (and marginal) in the 'conservative wrong' condition, $b=.04$, se $=.02,95 \% \mathrm{Cl}[-.00,0.09], Z=1.79$, $p=.07$, than in the 'politically neutral' condition, $b=.00$, se $=.01,95 \% \mathrm{Cl}[-.0 \mathrm{I}, 0.2], Z=0.44, p$ $=.66$, but note that wrongness judgments did not significantly mediate the effect of political orientation on free will attributions in either condition at traditional levels of statistical significance..

\section{Discussion}

In Study 6a, we tested the prediction that conservatives' greater attributions of free will would only be observed for events that they saw to be more morally wrong, and that these perceptions of wrongness would mediate the relationship between political orientation and free will attributions. Interestingly, despite having pre-tested items to be politically neutral in perceived moral wrongness, conservatives still showed a tendency to find the politically 
neutral items more morally wrong, supporting the view that conservatives moralize more than liberals. As predicted, higher political conservatism only predicted higher free will attributions for actions that were pre-tested to be perceived as more wrong by conservatives. We also found some suggestive, but not statistically significant, evidence that moral wrongness statistically mediated the relationship between more conservative political ideology and attributions of free will mainly in the 'conservative wrong' condition. In Study 6b we sought to enhance our confidence in these results by conducting a new pre-registered replication with a larger sample size.

\section{Method}

\section{Study 6b}

Open Science. Our design, hypotheses, and analysis plan were all pre-registered at the Open Science Framework. We report all measures, manipulations, and exclusions, and analysis code, and experiment materials are available for download from the OSF. This study received ethical approval from the IRB of the University of British Columbia ("Social Impacts of Emerging Technology"; Protocol \#H I8-02727).

Participants. 59I American participants completed the survey online via MTurk. Participants were excluded from data analysis if they took the survey more than once $(n=10)$ or failed one or both of two simple checks in which they had to select a certain scale-point in the FAD+ and then indicate at the end of the survey which event they did not answer questions about $(n=26)$. This left a final sample of 555 participants ( 280 female; $M_{a g e}=37$ years).

Design. This study was identical to Study 6a, except that we replaced some of the names of the protagonists to ensure they were matched in perceived age, warmth, and competence (Newman et al., 2018; see discussion in pre-test).

\section{Results}

First, we again looked at correlations of political conservativism with general free will belief. As in the previous studies, conservatism was significantly positively correlated with scores on the free will subscale of the FAD $+(r=.33, p<.001)$, with conservatives showing higher endorsement of both the more abstract $(r=.29, p<.001)$ and moral responsibility focused $(r=.34 p<.001)$ items of the scale.

Next, we checked whether political orientation was indeed associated with greater judgments of moral wrongness in the 'conservative wrong' condition. Here, political ideology was significantly associated with thinking the moral transgressions were more wrong $(r=.5 \mathrm{I}$, $p<.00 \mathrm{I})$, but in the matched condition with items pre-tested to be politically neutral, we found no correlation of political ideology with wrongness ratings $(r=.08, p=.21)$. Across participants, wrongness ratings were positively associated with free will attributions, both for the conservative wrong events $(r=.24, p<.001)$ and the politically neutral events $(r=.16, p$ $=.007)$.

We then turned to our key analyses and examined how morality condition $(-\mathrm{I}=$ politically neutral; I = conservative wrong) and participant political orientation (centered) interacted to predict attributions of free will. We observed a significant interaction of political ideology and morality condition, $b=.05, \mathrm{SE}=.02, t=2.7 \mathrm{I}, p=.007,95 \% \mathrm{Cl}[.0 \mathrm{I}, .08], \mathrm{R}_{2}=. \mathrm{II}$, semi-partial $r=0.1 \mathrm{I}$, whereby as predicted conservative ideology predicted more free will for 
the 'conservative wrong' items, $b=.09, \mathrm{SE}=.02, t=3.76, p<.00 \mathrm{I}, 95 \% \mathrm{Cl}[.04, .14], \mathrm{R}_{2}=.05$, semi-partial $r=.22$, but there was no difference in attributions of free will for the politically neutral events, $b=.00, S E=.02, t=0.0 \mathrm{I}, p=.99,95 \% \mathrm{Cl}[-.05, .05], \mathrm{R}_{2}=.00$, semi-partial $r=$ -.00 .

Finally, we observed significant moderated mediation, 95\% $\mathrm{Cl}[.04, .08]$, which we then probed by looking at the mediation of wrongness judgments within each condition $(10,000$ resamples). With this larger sample size, we found that wrongness judgments statistically significantly mediated the effect of more conservative political ideology on higher free will attributions in the 'conservative wrong' condition, $b=.04$, se $=.02,95 \% \mathrm{Cl}[.0 \mathrm{I}, .06], \mathrm{Z}=2.4 \mathrm{I}$, $p=.02$, but not in the 'politically neutral' condition, $b=.05$, se $=.00,95 \% \mathrm{Cl}[-.00, .0 \mathrm{I}], \mathrm{Z}=$ I. $15, p=.25$.

\section{Discussion}

In Study 6b, we conducted a pre-registered replication of Study 6a to test our prediction that conservatives' greater attributions of free will would only be observed for events that they saw to be more morally wrong, and that these perceptions of wrongness would mediate the relationship between political orientation and free will attributions. Confirming our predictions, political ideology only predicted free will attributions for actions pre-tested to be perceived as more wrong by conservatives, and the relationship between ideology and free will attributions was statistically mediated by perceptions of moral wrongness. Together, the results of Studies $6 \mathrm{a}$ and $6 \mathrm{~b}$ provide further support for our main hypothesis that the relationship between political ideology and free will beliefs can be at least partially linked to differential perceptions of moral wrongness and blameworthiness.

\section{Study 7}

It seems likely that political differences in attributions of free will (Study 3) are not reflecting some genuine disagreement about the basis of human freedom, given that these differences appear only for those events that are also judged as differentially morally wrong (Studies 4-6). Philosophically, the concept of free will should be domain-general and enduring: the deterministic laws of the universe are unlikely to be sensitive to the specific moral qualities of the action. Perhaps psychologically, though, people have heuristics about which actions require more or less free will (regardless of the philosophical coherence of such a stance). If this were the case, perhaps conservatives and liberals simply have different heuristics about which actions are more free, and higher perceptions of freedom cause stronger judgments of moral wrongness rather than the reverse motivated reasoning pattern we hypothesize (where moral assessments influence free will assessments). In our final set of studies, therefore, we wanted to conduct an even more direct test of the specifically motivated basis of political differences in attributions of free will by looking at whether liberals and conservatives would differentially attribute free will for the same action that differed only on who performed it so as to isolate motivations to blame. We do this across four studies, Studies 7a-7d. These are the only four studies we have conducted testing this interaction (i.e., there are no file drawer studies).

\section{Method}

\section{Study $7 a$}




\section{Open Science and Ethics Statement}

We report all measures, manipulations, and exclusions. Results, analysis code, and experiment materials are available for download at the OSF. This study, along with Studies $7 b$, $7 c$, and $7 d$, received ethical approval from the IRB of the University of British Columbia ("Social Impacts of Emerging Technology"; Protocol \#HI8-02727).

Participants. 600 American participants completed the survey online via MTurk. Participants were excluded from data analysis if they took the survey more than once $(n=I)$ or failed one or both of two simple checks in which they had to select a certain scale-point in the FAD+, and then correctly report at the end of the study what the protagonist in the vignette had done $(n=7)$. This left a final sample of 592 participants $(32 \mathrm{I}$ female; Mage $=38$ years).

Pre-Tests. We completed two pre-tests on MTurk to find events to use for Study 7a. In both pre-tests we only asked participant to rate wrongness and blameworthiness and not free will to avoid biasing item selection.

In our first pre-test $(n=249)$, we used six conventional moral violations and simply varied whether the actor was characteristically left- or right-wing (see materials on the OSF for the wording of all events and the corresponding results). For example, in one event we described to participants someone "who regularly smokes cigarettes indoors around his small child", and varied whether this person was "an activist who frequently protests against Wall Street" or "a wealthy Wall Street banker". In another event we described someone who was "recently arrested for driving while approximately two drinks over the legal driving limit", and varied whether this person "conducts research for a think tank and investigates how pervasive racism might explain disparate outcomes between racial groups" or "conducts research for a think tank and investigates genetic differences between races that might explain disparate outcomes between racial groups". Across these events, we failed to find significant interactions between participants' own political beliefs and the apparent political beliefs of the target: participants tended to rate the moral violation as equally wrong regardless of whether it was a left or right-wing person doing it. In other words, for these general behaviors, liberals and conservatives displayed no favoritism toward their own political ingroup members in terms of their moral evaluations. While pleasant from a normative perspective, this rendered these events unfit for our purposes in the main study.

To this end, we conducted a second pre-test $(n=300)$ in which we gave participants longer descriptions of moral violations that an actor performed in the context of achieving certain political goals (see materials on the OSF for the wording of all events and the corresponding results). This was done for two reasons: first, by giving more detailed information we hoped to make it less obvious to participants that we expected them to use the actor's political affiliation as a cue to wrongness; and second, to leverage partisan intergroup cognition that would plausibly make the moral violations more acceptable if it was done against "them", for "us". One item concerned using charity funds to buy a more expensive suit for oneself in the hopes this will impress donors for one's charity; one concerned blackmailing a town mayor about his use of prostitutes in order to get him to publicly support one's social movement; and one concerned a student who gets violent at a protest. As before, in this pre-test we measured only participants ratings of wrongness and blameworthiness, not 
free will, in order to avoid biasing our item selection. There was no significant interaction between participant political orientation and the political leanings of the target on ratings of wrongness for the first item, but there was for the second and third items, so these were the ones used in Study 7a.

Design. All participants read a short story about an immoral action performed by "Noah". In a between-subjects design, participants were randomly assigned to learn that Noah was either politically conservative or liberal and performed the action in the context of achieving these political ends. To increase confidence in the generalizability of our results, as an additional between-subjects factor we used two different events (violence at a protest and blackmail). As noted above, pre-testing revealed that in both events, the same action was perceived as differently wrong depending on the participant's political beliefs and the beliefs of the actor.

In the first story, participants read that Noah was a college student who gets caught up in a student protest and "In the heat of the moment, throws a glass bottle at the other protestor - though luckily, it didn't hit them”. For half of our participants, Noah was described as supporting "the Antifa movement - a left-wing protest movement that often gets into violent clashes with more right-wing protesters". The other half of participants read instead that Noah supported "the Patriot Movement - a right-wing protest movement that often gets into violent clashes with more left-wing protesters." Full text can be seen at the OSF.

In the second story, participants read that Noah was the local chapter head of a political group who blackmails the mayor of his town in order to obtain support for his movement. For half of our participants, Noah was described was leading the local "Black Lives Matter" movement, and blackmails the mayor into "calling for stricter punishment for police who kill black people". For the other half, Noah was described as leading the local "Blue Lives Matter" and instead blackmails the mayor into "calling for stricter punishment for people that kill law enforcement officers".

After reading the story, participants were asked to rate the degree of free will, wrongness, and blameworthiness associated with each described event (as in Studies 3-6). The order of the five dependent variables was randomized, and at the end participants completed the free will subscale of the FAD+.

As anticipated, results were the same for both stories and so we report our main analyses combining across the two. Full results for each individually can be seen at the OSF.

\section{Results}

First, we looked at correlations of political conservativism with general free will belief. As in the previous studies, conservatism was significantly positively correlated with scores on the free will subscale of the FAD+ $(r=.25, p<.001)$, with conservatives showing higher endorsement of both the more abstract $(r=.23, p<.001)$ and moral responsibility focused $(r$ $=.23 p<.00 \mathrm{I}$ ) items of the scale.

Second, we confirmed that participants did perceive the moral violation to be more wrong when the agent and the agent's actions opposed their own political group than when the agent and the agent's actions supported their own political group. A regression analysis revealed a significant interaction between the agent's political position (-I right-wing target, + I left-wing target) and participant's own political orientation (centered) on ratings of moral 
wrongness, $b=.21$, SE $=.03, t=6.37, p<.001,95 \% \mathrm{Cl}[.15, .28], \mathrm{R}_{2}=0.07$, semi-partial $r=0.25$. Whereas participant conservatism predicted thinking the left-wing agent performed a more morally wrong action, $b=.22, \mathrm{SE}=.05, t=4.60, p<.00 \mathrm{I}, 95 \% \mathrm{Cl}[. \mathrm{I} 2, .3 \mathrm{I}], \mathrm{R}_{2}=0.07$, semipartial $r=0.26$, political liberalism predicted thinking the right-wing agent performed a more morally wrong action, $b=-.20, S E=.05, t=-4.4 \mathrm{I}, p<.00 \mathrm{I}, 95 \% \mathrm{Cl}[-.29,-. \mathrm{II}], \mathrm{R}_{2}=0.06$, semipartial $r=-0.25$.

Finally, we turned to our main analysis of whether participants attributed differential free will for the same action depending on their own political beliefs (centered) and the political beliefs of the actor (-I right-wing target, + I left-wing target). The interaction did not reach statistical significance, $b=.03, S E=.02, t=1.70, p=.089,95 \% \mathrm{Cl}[-.0 \mathrm{I}, .07], \mathrm{R}_{2}=0.0 \mathrm{I}$, semi-partial $r=0.07$, and simple effects indicated that political conservatism predicted lower attributions of free will towards the right-wing target, $b=-.07, S E=.03, t=-2.38, p=.018,95 \%$ $\mathrm{Cl}[-.12,-.01] \mathrm{R}_{2}=0.02$, semi-partial $r=-0.14$, but not the left-wing target $b=.00, \mathrm{SE}=.03, t$ $=0.12, p=.91,95 \% \mathrm{Cl}[-.05, .06] \mathrm{R}_{2}=0.00$, semi-partial $r=0.01$. Probing this further by looking at attributions of free will by self-identified Republican $(n=160)$ and Democrat $(n=249)$ participants, we found a significant interaction of participant political affiliation and the agent's political beliefs on attributions of free will, $F(\mathrm{I}, 405)=3.99, p=.047, p=.0 \mathrm{I}$, partial $\eta 2=.0 \mathrm{I}$. Simple effects results revealed that whereas Democrats attributed marginally more free will to the right-wing than left-wing agent, $t(246.83)=-1.80, p=.074, d=0.23$, Republicans, however, did not significantly differ in the degree of free will they attributed to both agents, $t(I 58)=1.12, p=.266, d=0.18$

\section{Discussion}

In Study 7a, we took a different approach to exploring the motivated basis of free will belief by looking at whether participants' political orientation might lead to differential attributions of free will for the same action depending on who performed it. Using two different moral violations that yielded the same results, we found tentative - but weak evidence in support of our predictions: there was the suggestion of differential free will attributions for the same action depending on participants' own political beliefs, though this was not significant.

Though these results appear to only weakly align with the findings of the previous studies, ceiling effects in Study 7a's measures of both moral violation and free will may have limited our ability to properly test our hypothesis. Regardless of the actor's political beliefs, both Republican and Democrat participants thought the moral violation very wrong (all means $>5.8$ on a I-7 scale) and - more problematically - attributed high free will to both actors (all means $>6.19$ on a I-7 scale). Previous work has shown that because people like to see themselves as fair and objective judges, motivated reasoning is most pronounced in situations where plausibility constraints are loose and ambiguous (Ditto \& Boardman, 1995; Ditto \& Lopez, 1992; Dunning et al., 1995). The events described clear moral violations, and with such high free attributions across the board, there might have been little room for motivated blame to selectively increase free will attributions for the politically-opposing agent. Given this, in Study $7 b$ we sought to run the study again, but with events that were more morally ambiguous.

\section{Study 7b}




\section{Method}

\section{Open Science}

We report all measures, manipulations, and exclusions. Results, analysis code, and experiment materials are available for download from the OSF.

Participants. 600 American participants completed the survey online via MTurk. Participants were excluded from data analysis if they took the survey more than once $(n=0)$ or failed one or both of two simple checks in which they had to select a certain scale-point in the FAD+, and then correctly report at the end of the study what the protagonist in the vignette had done $(n=34)$. This left a final sample of 566 participants $\left(274\right.$ female; $M_{\text {age }}=38$ years).

Design. This study had the same design as Study 7a, where again participants read a story describing a moral violation and in a between-subjects design we manipulated whether the actor was politically right-wing or left-wing and performed the action in the context of achieving those political ends. Again, to increase confidence in the generalizability of our results, as an additional between-subjects factor, we used two different events, but sought to use scenarios more ambiguously immoral than in Study 7a (staying silent about disruption at a protest; not retracting a magazine expose based on an unreliable source). Pre-testing (see details at OSF) revealed that in both events, the same action was perceived as differently wrong depending on the participant's political beliefs and the beliefs of the actor (and that overall, the items were rated as less wrong than the items in Study 7a).

In the first story, participants read about "Anna": an editor and journalist who publishes a widely-discussed and influential expose about a sex scandal amongst the highest members of a political party. Participants were told that "A few months after this expose was released, Anna learns about serious problems with her source which makes her doubt whether they are telling the truth. The source is adamant they are telling the truth, but Anna has found that they have made false accusations in the past. Usually Anna would be required to publish a retraction, but because the evidence of lying is not conclusive, she does not issue a correction.". For half of our participants, Anna was described as being the editor of a "prominent left-wing online journal known for regularly and strongly attacking the Republican party", who publishes an expose about a sex scandal in the Republican party. For the other half, Anna was described as being the editor of a "prominent right-wing online journal" who publishes an expose about a sex scandal in the Democratic party.

In the second story, participants read about Noah, a politically active college student who is peacefully protesting a visit by a controversial speaker. During the protest, Noah observes a fellow student "cut the sound for the speaker, meaning that no-one can hear the talk and it has to be called off". Participants were told that the police want to find and prosecute the activist "to make an example of them, drawing attention to what they see as a rising problem of right/left-wing activists", but "When the police come and question Noah, he says that he did not see who cut the sound". Half of our participants were told that both Noah and the activist who cut the sound were members of the "Young Republicans Society" and were protesting a controversial left-wing speaker.The other half were told that Noah and the activist were members of the "Young Democrats Society" and were protesting a right-wing speaker (see full wording on the OSF). 
The dependent measures were the same as in Study $7 \mathrm{a}$ and because results were again the same for both stories, we report our main analyses combining across the two (see OSF for results for event separately).

\section{Results}

First, we looked at correlations of political conservativism with general free will belief. As in the previous studies, conservatism was significantly positively correlated with scores on the free will subscale of the FAD+ $(r=.16, p<.001)$, with conservatives showing higher endorsement of both the more abstract $(r=.29, p<.001)$ and moral responsibility focused $(r$ $=.26 p<.00 \mathrm{I})$ items of the scale.

Second, we confirmed that participants did perceive the moral violation to be more wrong when the target opposed rather than supported their own political interests. A regression analysis revealed a significant interaction between the agent's political position (-I right-wing target, + I left-wing target) and participant's own political orientation (centered) on ratings of moral wrongness, $b=.27, \mathrm{SE}=.04, t=6.82, p<.00 \mathrm{I}, 95 \% \mathrm{Cl}[.19, .35], \mathrm{R}_{2}=0.13$, semi-partial $r=0.27$. Whereas participant conservatism predicted thinking the left-wing agent performed a more morally wrong action, $b=.50, S E=.05, t=9.3 \mathrm{I}, p<.00 \mathrm{I}, 95 \% \mathrm{Cl}[.39, .60]$, $R_{2}=0.23$, semi-partial $r=0.48$, despite our pre-testing, we found no effect of political orientation on wrongness ratings of the conservative target, $b=-.04, S E=.06, t=-0.72, p$ $=.48,95 \% \mathrm{Cl}[-.16,-.07] \mathrm{R}_{2}=0.00$, semi-partial $r=-0.04$.

Finally, we turned to our main analysis of whether participants attributed differential free will for the same action depending on their own political beliefs (centered) and the political beliefs of the actor (-I right-wing target, $+I$ left-wing target). We observed a significant interaction, $b=.09, \mathrm{SE}=.02, t=3.85, p<.001,95 \% \mathrm{Cl}[.05, .14], \mathrm{R}_{2}=0.03$, semi-partial $r=$ 0.16 , where simple effects indicated that political conservatism predicted greater attributions of free will towards the left-wing target, $b=.13, \mathrm{SE}=.03, t=3.90, p<.001,95 \% \mathrm{Cl}[.06, .19]$, $R_{2}=0.05$, semi-partial $r=0.23$, but there was no significant effect of participant political orientation on attributions of free will towards the right-wing target (though the direction of results was as expected), $b=-.06, S E=.04, t=0.1 \mathrm{I}, p=.1 \mathrm{I}, 95 \% \mathrm{Cl}[-.13, .0 \mathrm{I}], \mathrm{R}_{2}=0.0 \mathrm{I}$, semipartial $r=-0.10$ (see Figure 3). Again probing this further by looking at attributions of free will by self-identified Republican $(n=140)$ and Democrat $(n=235)$ participants, we found a significant interaction of participant political affiliation and the agent's political beliefs on attributions of free will, $F(I, 37 I)=7.46, p=.007$, partial $\eta 2=.02$. Simple effects results revealed that Democrats attributed significantly more free will to the right-wing than left-wing agent, $t(232.98)=2.17, p=.031, d=0.28$, and there was a trend for Republican participants to attribute more free will to the left-wing agent than the right wing one, though this was only marginally significant, $t(\mid \mathrm{I} 28.05)=-I .80 p=.074, d=0.30$ 


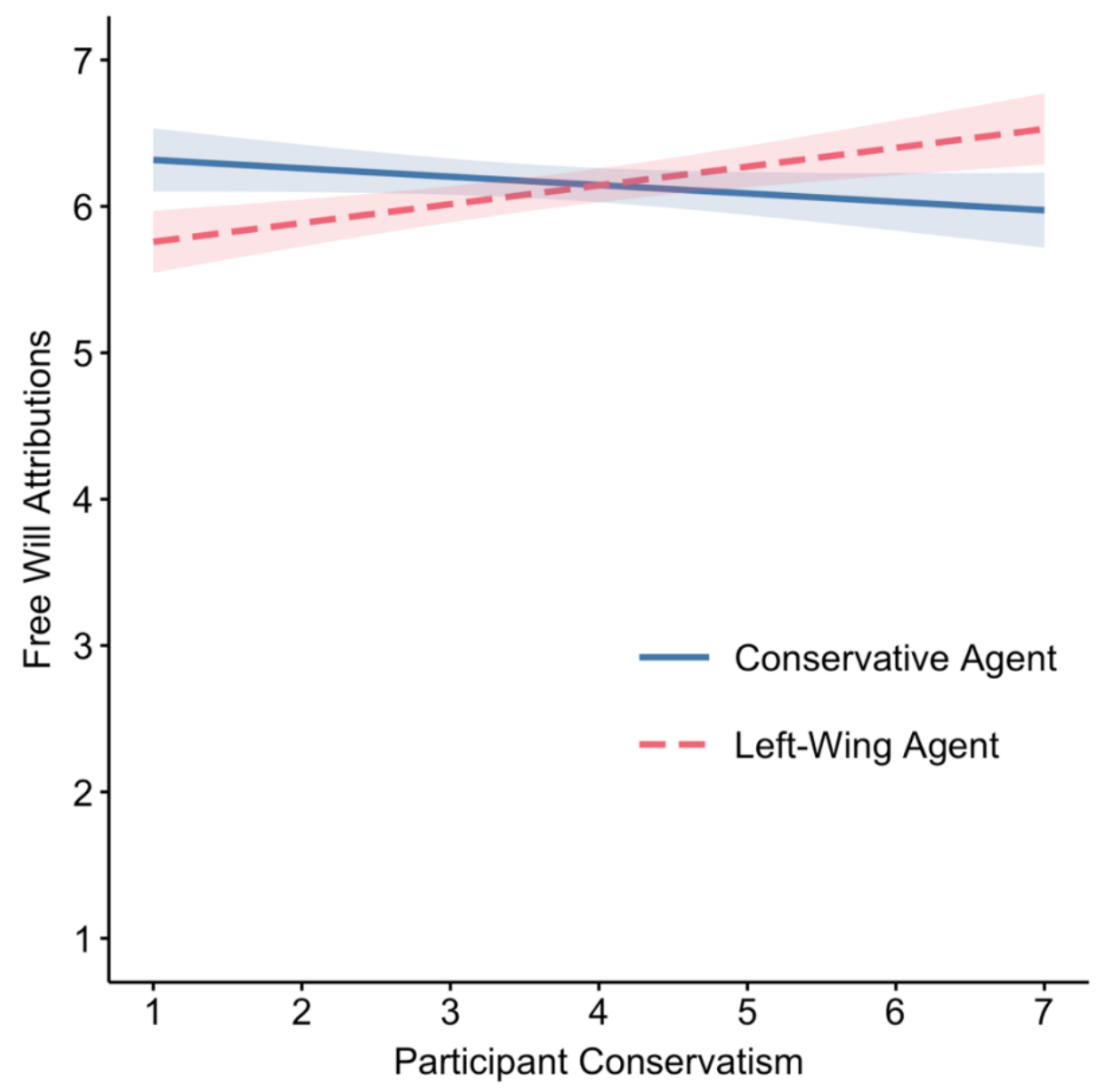

Figure 3. Attributions of free will as a function of participant political ideology and the political beliefs of the actor (Study 7b).

\section{Discussion}

In Study $7 b$ we found further evidence for the motivated basis of free will belief by showing that participants attribute different levels of free will for the same action depending on whether someone shares or disagrees with their own political stance. Note, however, that our claim is not that political conservatives and liberals will always differentially attribute free will for the same event depending on who does it, just as we are not claiming that political conservatives and liberals will always make in-group favouring biased moral judgments (indeed, our first pre-test for Study 7a shows that this is not the case). Instead, we arguing that where there is a motivation to differentially blame the target that emerges in biased ratings of moral wrongness, there will typically be a motivation to differentially attribute free will too. To confirm our results, in Study 7c we opted to conduct our second pre-registered replication of this series.

\section{Method}

\section{Study 7c}

\section{Open Science}

Our design, hypotheses, and analysis plan were all pre-registered at the Open Science Framework. We report all measures, manipulations, and exclusions, and analysis code, and experiment materials are available for download from the OSF. 
Participants. 595 American participants completed the survey online via MTurk. Participants were excluded from data analysis if they took the survey more than once $(n=0)$ or failed one or both of two simple checks in which they had to select a certain scale-point in the FAD+, and then correctly report at the end of the study what the protagonist in the vignette had done $(n=58)$. This left a final sample of 537 participants $\left(255\right.$ female; $M_{\text {age }}=38$ years).

Design. This study had the same design as Study $7 \mathrm{~b}$.

\section{Results}

First, we looked at correlations of political conservativism with general free will belief. As in the previous studies, conservatism was significantly positively correlated with scores on the free will subscale of the FAD+ $(r=.28, p<.001)$, with conservatives showing higher endorsement of both the more abstract $(r=.26, p<.001)$ and moral responsibility focused $(r$ $=.25 p<.001$ ) items of the scale.

Second, we confirmed that participants did perceive the moral violation to be more wrong when the agent opposed rather than aligned with their own political interests. A regression analysis revealed a significant interaction between the agent's political position (-I right-wing target, + I left-wing target) and participant's own political orientation (centered) on ratings of moral wrongness, $b=.25, \mathrm{SE}=.04, t=5.86, p<.00 \mathrm{I}, 95 \% \mathrm{Cl}[.17, .33], \mathrm{R}_{2}=0.09$, semi-partial $r=0.24$. Whereas participant conservatism predicted thinking the left-wing agent performed a more morally wrong action, $b=.4 \mathrm{I}, \mathrm{SE}=.06, t=6.87, p<.00 \mathrm{I}, 95 \% \mathrm{Cl}[.30, .53]$, $R_{2}=0.15$, semi-partial $r=0.39$ despite our pre-testing (but as in Study $7 b$ ), we found no effect of political orientation on wrongness ratings of the conservative target, $b=-.08, S E=.06, t=$ $-1.39, p=.17,95 \% \mathrm{Cl}[-.02,-.03], \mathrm{R}_{2}=0.0 \mathrm{I}$, semi-partial $r=-0.08$.

Finally, we turned to our main analysis of whether participants attributed differential free will for the same action depending on their own political beliefs (centered) and the political beliefs of the actor (-I right-wing target, + I left-wing target). Despite the fact that this was a direct registered replication on Study $7 b$ in which we found a significant interaction, the interaction here was non-significant, $b=.0 \mathrm{I}, \mathrm{SE}=.03, t=0.32, p=.75,95 \% \mathrm{Cl}[-.06, .08], \mathrm{R}_{2}=$ 0.00 , semi-partial $r=0.03$. Surprisingly, political conservatism did not predict greater attributions of free will towards either the left-wing target, $b=.0 \mathrm{I}, \mathrm{SE}=.03, t=0.32, p=.75$, $95 \% \mathrm{Cl}[-.06, .08], \mathrm{R}_{2}=0.00$, semi-partial $\mathrm{r}=0.02$, or the right-wing target, $b=-.02, \mathrm{SE}=.04, t$ $=-0.58, p=.56,95 \% \mathrm{Cl}[-.09, .05], \mathrm{R}_{2}=0.00$, semi-partial $r=-0.04$. Similarly, when looking at attributions of free will by self-identified Republican $(n=|3|)$ and Democrat $(n=232)$ participants, we found no interaction of participant political affiliation and the agent's political beliefs on attributions of free will, $F(I, 359)=0.59, p=.44$, partial $\eta 2=.00$.

\section{Discussion}

In Study 7c we conducted our second pre-registered replication in this package of studies. We sought to replicate our finding from Study $7 \mathrm{~b}$ that participants attribute different levels of free will for the same action depending on whether someone shares or disagrees with their own political stance. Surprisingly, our key interaction was not significant - despite using the same materials, sample recruitment, participant exclusion rules, and data analysis.

How to explain this? One possibility is that on the I-7 scale, we had high free will attributions across the board (>5.5), potentially leading to ceiling effects. Another possibility is 
the time we ran the study. The data for Study 7c were collected between I $2 \mathrm{am}$ and 6am PST (3am to 9am EST). With our restriction that participants needed to be from the U.S. to complete the study, this meant that participants were completing the study in the middle of the night. Despite employing the same attention and comprehension checks used across the studies in this paper, it is possible that participants were still not attending as thoroughly as they would have been if they hadn't been doing it in the middle of the night. Perhaps the null results in Study 7c were just a fluke, potentially influenced by ceiling effects and/or the unusual time of data collection. Or perhaps it was the significant effect in Study $7 b$ that was the fluke, and that there was no real effect to find at all. In Study $7 d$ we sought conduct one final preregistered replication to settle the matter, making sure to collect the data during the day while also using a larger sample size and increasing the scale variance from I-7 to 0-100.

\section{Study 7d}

\section{Method}

\section{Open Science}

Our design, hypotheses, and analysis plan were all pre-registered at the Open Science Framework. As for all studies in this paper, we report all measures, manipulations, and exclusions, and analysis code, and experiment materials are available for download from the OSF.

Participants. We recruited 900 American participants via MTurk, 884 of whom completed the survey. Participants were excluded from data analysis if they took the survey more than once $(n=1)$ or failed one or both of two simple checks in which they had to select a certain scale-point in the FAD+, and then correctly report at the end of the study what the protagonist in the vignette had done $(n=68)$. This left a final sample of 815 participants (478 female; Mage $_{\text {= }} 37$ years).

Design. This study had the same design as Study $7 b$ and 7c, with the exception that, in Study 7d, the dependent measures were on a 0-100 scale instead of $1-7$ scale, and we also included an additional question about how severely participants thought the agent should be punished for their action.

\section{Results}

First, we looked at correlations of political conservativism with general free will belief. As in the previous studies, conservatism was significantly positively correlated with scores on the free will subscale of the FAD+ $(r=.34, p<.001)$, with conservatives showing higher endorsement of both the more abstract $(r=.30, p<.001)$ and moral responsibility focused $(r$ $=.32 p<.001$ ) items of the scale.

Second, we confirmed that participants did perceive the moral violation as more wrong when the agent aligned with — rather than opposed - their own political interests. A regression analysis revealed a significant interaction between the agent's political position (-I right-wing target, + I left-wing target) and participant's own political orientation (centered) on ratings of moral wrongness, $b=5.33, \mathrm{SE}=.58, t=9.12, p<.00 \mathrm{I}, 95 \% \mathrm{Cl}[.4 .19,6.48], \mathrm{R}_{2}=0.13$, semipartial $r=0.30$. Participant conservatism positively predicted thinking the left-wing agent performed a more morally wrong action, $b=7.82, \mathrm{SE}=.86, t=9.07, p<.00 \mathrm{I}, 95 \% \mathrm{Cl}[6 . \mathrm{I} 2$, $9.5 \mathrm{I}], R_{2}=0.17$, semi-partial $r=0.4 \mathrm{I}$, and negatively predicted wrongness ratings for the same 
action when performed by a right-wing agent, $b=-2.85, S E=.79, t=-3.60, p<.00 \mathrm{I}, 95 \% \mathrm{Cl}[-$ $4.41,-1.29], R_{2}=0.03$, semi-partial $r=-0.17$.

Finally, we turned to our main analysis of whether participants attributed differential free will for the same action depending on their own political beliefs (centered) and the political beliefs and interests of the actor ( - I right-wing target, + I left-wing target). We observed a significant interaction, $b=1.45, S E=.035, t=4.15, p<.001,95 \% \mathrm{Cl}[.77,2.14], \mathrm{R}_{2}$ $=0.03$, semi-partial $r=0.14$. Simple effects indicated that political conservatism negatively predicted attributions of free will towards the right-wing target, $b=-2.03, S E=.48, t=-4.27, p$ $<.00 \mathrm{I}, 95 \% \mathrm{Cl}$ [-2.97, - I.I0], $\mathrm{R}_{2}=0.04$, semi-partial $r=-0.2 \mathrm{I}$. The effect of participant conservatism on free will attributions for the left-wing agent was not significant, though the direction of results was as expected: $b=0.88, S E=.52, t=1.70, p=.090,95 \% \mathrm{Cl}[-.14,1.89]$, $R_{2}=0.01$, semi-partial $r=0.08$ (see Figure 4). Again probing this further by looking at attributions of free will by self-identified Republican $(n=180)$ and Democrat $(n=354)$ participants, we found a significant interaction of participant political affiliation and the agent's political beliefs on attributions of free will, $F(I, 530)=6.80, p=.009$, partial $\eta 2=.0 \mathrm{I}$. Simple effects results revealed that Republicans attributed significantly more free will to the left-wing agent, $t(176.46)=2.35, p=.020, d=0.35$, but Democrats did not differ in attributions depending on the agent, $t(345.74)=-1.18, p=.24, d=-0.13$.

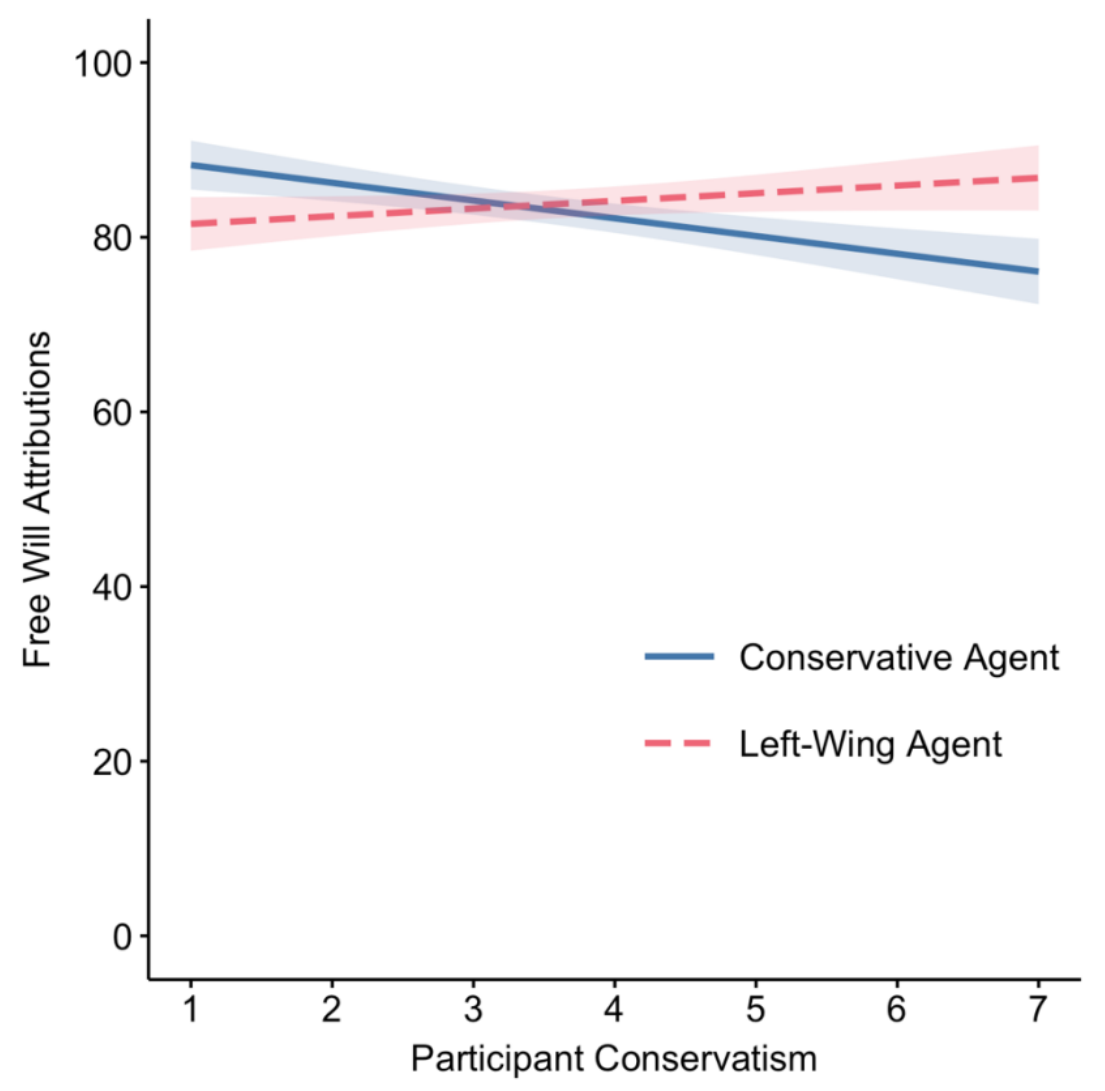

Figure 4. Attributions of free will as a function of participant political ideology and the political beliefs of the actor (Study 7d).

\section{Discussion}


In Study 7d we conducted a final pre-registered replication in attempt to clarify inconsistencies in our findings in Study 7a-c. With a larger sample size and increasing the scale variance from 1-7 to 0-100, we replicated the significant interaction found in Study 7b: participants attributed different levels of free will for the same action depending on whether the target shared or disagreed with their own political stance.

\section{Study 7e: Meta-analysis of Studies 7a-7d}

Because the effect sizes for the interaction between ideology and the experimental manipulation varied somewhat across Studies 7a-7d, we concluded with mini-meta-analyses of the interaction effect for these four studies. As noted in the introduction, we have reported in the manuscript all the studies we have run testing this interaction.

\section{Method}

We used procedures outlined by Goh and colleagues (2016) for conducting mini-meta-analyses on the four interaction effects between ideology and the experimental manipulation in Studies 7a-7d. Because the methods used across studies were very similar (in which case fixed effects, which weights by sample size, might be preferred), but not quite identical (in which case random effects, which treats all effects equally, might be preferred), we report both fixed and random effects, though note the random effects approach is very conservative with only four effect sizes (Goh et al., 2016). We used semipartial $r$ s as estimates of the effect size for the interaction terms. For fixed effects, the four rs were Fisher's $Z$ transformed to $r_{z} s$, which were then weighted and averaged using the following formula: Weighted $\overline{r_{Z}}=\Sigma\left([N-3] r_{Z}\right) / \Sigma(N-3)$. The weighted $\overline{r_{Z}}$ was then converted back to a Pearson's $r$ correlation for presentation. To determine statistical significance, we utilized the Stouffer's $Z$ test, in which the $p$ values for each interaction effect were converted to $Z$ s, combined using the following formula: $Z_{\text {combined }}=\Sigma Z / \operatorname{sqrt}(k)$, and then converted back to ps for presentation. For random effects, we conducted a single sample $t$-test of the semipartial $r s$.

\section{Results}

For both random effects $(r=.101, p=.048)$ and fixed effects $(r=.106, p$ $<.000 \mathrm{I}$ ), there were small but statistically significant effects of the interaction between ideology and the experimental manipulation on free will attributions.

\section{Discussion}

Though we found slightly different results across Studies 7a-7d, when analyzed together in a meta-analysis, these studies provided evidence for the existence of the hypothesized interaction. Thus, free will attributions for identical actions, vary as a function of whether those actions oppose or align with one's own political interests. In our view, these results provide the most compelling support for our contention that differences in perceptions of free will between liberals and conservatives reflect varying motivations to blame rather than principled and consistent beliefs about human freedom and control.

\section{General Discussion}

Personal responsibility and autonomy feature heavily in conservative ideologies (Reagan, 1968; Thatcher, 198I), and recent evidence has suggested that conservatives exhibit greater belief in free will than liberals (Carey \& Paulhus, 20I3). In this paper we sought to test 
the hypothesis that political differences in free will belief do not reflect some genuine principled disagreement about the metaphysical nature of human freedom, but rather are largely explicable through motivated reasoning. Previous work has shown that, in general, free will beliefs are motivated by desires to punish others and to justify holding them morally responsible (Clark et al., 2014, 2017, 2018, 2019), and in this paper we tested whether the reason that conservatives tend to attribute more free will is because they have a stronger tendency to moralize, perceiving a wider spectrum of transgressions for which moral responsibility must be assigned and moral blame attributed.

\section{Overview of Findings}

In Study I, we directly tested our background assumption that political conservatives have a stronger tendency to moralize (though this does not, of course, mean that conservatives always see things as more wrong than liberals: see Study 5). Meta-analysing five new studies drawn from a variety of populations (total $n=308,499$ ), we show that political ideology was consistently associated with moralization. Political conservatism was associated with greater wrongness judgments in the Moral Foundations Questionnaire (Study la); with perceiving minor everyday moral violations to be more wrong (Study Ib); with judging a variety of traits as more necessary for someone to be a morally good person (Study Ic); with perceiving someone as a bad person based on an image of their face (Study Id); and as rating personality traits as more morally bad (Study le).

In Study 2, we turned to investigate our main question of political differences in free will belief. Using a large sample of yourmorals.org data $(n=14,707)$ we looked at the relationship among political ideology, free will belief, and moralization. Replicating previous work (Carey \& Paulhus, 2013), we found that political conservatism was indeed associated with a greater belief in abstract free will. As would be expected if this relationship arises partially from moralization, we found that beliefs that people are morally responsible for their bad behaviors statistically mediated the relationship between more conservative ideology and stronger beliefs in free will.

In Study 3, we turned away from reports of general, abstract belief in free will to look at attributions of free will for specific events. More specifically, we wanted to look at attributions of free will for both positive and negative events. We found that political ideology predicted free will attributions overall, and there was an indication that this relationship was stronger for negative than positive events. Conceptually, the capacity for free will should hold whether one experiences good or bad outcomes, and so if ideology is genuinely related to an abstract belief in free will, there should have been no difference depending on the valence of the outcomes. The fact that conservatism predicted higher free will attributions mainly for negative events is consistent with the claim that free will attributions are not, or at least not solely, reflecting some dispositional variance in a belief in human autonomy, but a more basic, social psychological phenomenon - likely one relating to blame.

In Study 4, we considered that if political conservatives are more likely to attribute free will because they see more things as morally wrong, this association should not be observed when looking at attributions of free will for specific events that are perceived as equally (im)moral for liberals and conservatives. Using a range of both moral and non-moral, and positive and negative events that were pre-tested to be equally matched in morality and 
valence amongst political liberals and conservatives, we found no relationship between political ideology and free will attributions. Again, these results were consistent with our theory that differences in conservatives' and liberals' perceptions of free will may be partially due to differences in moralization, rather than representing any generalized, abstract belief that human behaviors are freely chosen.

In Study 5, we tested whether - if the typical tendency for conservatives to endorse stronger beliefs in free will is due to blame motives - this association would be reversed for those less frequent events that conservatives perceive as less morally wrong than their liberal counterparts. Whereas conservatives again reported higher general, abstract belief in free will as measured by the FAD+, when using events that liberals saw as more morally wrong than conservatives, it was liberals that attributed more free will.

In Study 6, we tested the prediction that conservatives' greater attributions of free will would only be observed for events that they saw to be more morally wrong, and that these perceptions of wrongness would mediate the relationship between political orientation and free will attributions. In two studies - an initial one (Study 6a), and then a pre-registered replication with a larger sample size (Study 6b) - we confirmed our predictions, showing that political ideology only predicted free will attributions for actions that pre-tested to be perceived as more wrong by conservatives, and this relationship was statistically mediated by perceptions of moral wrongness. Again, this supports our contention that political differences in free will beliefs are linked to differential perceptions of moral wrongness and blameworthiness.

In Study 7, we took a different approach. Instead of manipulating the moral content of the event and then looking at how political ideology is associated with free attributions, we looked at whether liberals and conservatives would differentially attribute free will for the same action depending on who performed it. In Study 7a, we used two different moral violations (violence at a protest; blackmail) and told participants that the action was performed by a leftwing or right-wing agent. Across both events we found tentative - but weak - evidence in support of our predictions: there was the suggestion of differential free will attributions for the same action depending on participants' own political beliefs, though this was not significant. Recognizing that this could have been because the events described clear and intentional moral violations, whereas motivated reasoning is most pronounced in situations where plausibility constraints are loose and ambiguous, in Study $7 \mathrm{~b}$ we used the same design but with events that were more morally ambiguous (staying silent about disruption at a protest; not retracting a magazine expose based on an unreliable source). Confirming predictions, we found that participants attributed different levels of free will for the same action depending on whether the actor shared or disagreed with their own political stance. To confirm our results, in Study 7c we conducted a pre-registered replication. Surprisingly, our key interaction was not significant - despite using the same materials, sample recruitment, participant exclusion rules, and data analysis. Because this could have been caused by technical problems, two months later we ran a second pre-registered replication for Study $7 \mathrm{~d}$. With a larger sample size and an increase in the scale variance from I-7 to $0-100$, we replicated the significant interaction as predicted, showing that participants attributed different levels of free will for the same action depending on whether the target shared or disagreed with their own political stance. Finally, 
because the effect sizes for the interaction between ideology and the experimental manipulation varied across Studies 7a-d, we concluded with mini-meta-analyses of the interaction effect for these four studies. These showed small but statistically significant effects of the interaction between ideology and the experimental manipulation on free will attributions.

Together, these I 4 studies paint a picture whereby conservatives' comparatively strong free will beliefs are linked to a desire to hold others accountable for transgressions, and not merely reflective of a generalized and abstract metaphysical belief concerning the nature of human agency. These results are consistent with our theory that the relationship between political ideology and free will beliefs can be at least partially explained as a manifestation of motivated cognition: People endorse the idea of free will in order to justify their desire to blame others for moral wrongdoing (Clark et al., 2014, 2017, 2019) with conservatives reporting higher free will beliefs in part because they find a wider spectrum of issues to be more morally wrong. Thus, to understand apparent differences in free will belief we need not appeal to some special kind of "Republican Brain" (Mooney, 20I2) that differs from that of liberals on metaphysical beliefs about human autonomy. Instead where we do see differences in free will beliefs, they are derived from more basic social cognitive processes that are shared regardless of the political party one votes for, and more basic differences in moralization.

\section{Limitations}

There are, as with any project, certain limitations to our analysis here. First, it is important to note that whereas we have focused on how moralization can help explain the relationship between political ideology and free will beliefs, we do believe that the causal relationships between our variables of interest (political ideology, free will beliefs, and moralization) are likely complex and involve feedback loops. There is already debate in the field as to whether it makes more sense to conceptualise political orientation as being caused by, or following from, different moral intuitions. Whereas some scholars have argued that moralization tendencies and a broader moral domain can explain why people are attracted to particular political ideologies (e.g., Graham et al., 2009; Haidt, 2012), others have argued for the opposite casual direction whereby individual differences associated with ideological beliefs relating to system justification and social dominance orientation are what explains differences in the moral domain (e.g., Hatemi et al., 2019; Kugler et al., 20I4; K. B. Smith et al., 20I7). Similarly, we have treated political ideology as a predictor variable and free will attributions as an outcome variable, but differences in perceived freedom likely lead to particular ideological views as well. We have little doubt that all three of our variables influence and reinforce one another. What we aimed to test here is whether experimentally manipulating differences in blame desires between ideological groups can generate (or eliminate) differences in free will attributions. We found consistent support for this, and thus, we think that this causal pattern does exist, but it does not preclude other causal patterns from also existing. It is thus possible that conservatives have proclivities for free will beliefs above and beyond moralization tendencies, and an individual's moralizing could lead to a more conservative ideology that further exaggerates their moralizing-related affinity for free-will beliefs. Investigating the possible feedback loops will be an interesting direction for future research. That said, we do believe that the model we have focused on here (political orientation predicts free will 
attributions partially through increased moralization) is likely to be a particularly promising approach. Whereas there is a large body of evidence showing substantial stability and consistency in ideological beliefs (e.g., Jost, 2006; Jost et al., 2008), along with significant heritability (Hatemi et al., 20I4) and distinct neurocognitive correlates (Amodio et al., 2007), we are aware of no such evidence establishing the stability, consistency, and heritability of free will beliefs. Indeed, our own results here show that while conservatives are consistently likely to report a higher abstract belief in free will, their specific attributions of free will are deeply context-dependent and susceptible to motivated cognition.

Second, there is room for debate about how precisely free will attributions should be conceptualised, and especially whether particpants' ratings of responsibility should be included in our measure of overall free will attributions. In line with previous empirical work in this area, we have used the term "free will" to refer to an autonomous choice of action that a person performs in the absence of substantial internal and external constraints (Baumeister \& Monroe, 20I4; Paulhus \& Carey, 20II), where this ability to choose renders one morally responsible for their actions (Nichols, 2007; Nichols \& Knobe, 2007). In short, scholars and laypeople alike appear concerned with free will primarily because they are concerned with responsibility. And we are interested in motivated attributions of free will precisely because attributions of free will create a sense of responsibility, which is relevant to many political disagreements along both economic and social dimensions. It is for these reasons that our pre-registered composite measure of free will attributions included a question about how responsible participants judged the actor to be, in addition to questions about the actor's control, free will, and ability to choose otherwise. For scholars who would restrict conceptions of free will to those related to choice, control, and freedom (and not responsibility), we crosschecked our main results without the responsibility item and this did not impact the statistical significance of any of our main results. Thus the pattern of results here applies to the lay conception of free will regardless of whether that includes responsibility or not.

Third, our analysis here examines how manipulating desires to blame increases or decreases ideological differences in tendencies to attribute free will to individual actors, yet they are only suggestive of potential influences on persistent individual differences in free will beliefs. We provided evidence that (I) conservatism is associated with higher beliefs in free will, (2) conservatism is associated with moralizing, and (3) increasing desires to blame increases attributions of free will to individual actors. It is therefore theoretically and empirically plausible that conservatives, experiencing stronger blame desires in their everyday life, might come to believe more in free will for this reason, but the present work cannot confirm this definitively. We hope the present results might inspire future work to seek ways of exploring the causes of persistent individual differences among liberals and conservatives in their free will beliefs, and particularly to explore the impact of persistent desires to blame.

Fourth, our research here involved attributions of free will for hypothetical (albeit realistic) and not actual events. This was a deliberate decision to allow us to cleanly manipulate perceived moral wrongness and control for the information that participants received. Though we judged this as preferable to studying current real-world situations in which participants have different levels of knowledge and investment, it could be interesting for future work to 
consider the relationship between political ideology, moralization, and attributions of free will in real-world contexts.

Finally, it should be noted that our experimental research (Studies 3-7) has focused on data from U.S. participants via MTurk.A key strength of MTurk is that it yields data that are more representative than those from traditional student samples - especially on the dimensions of age and political ideology (Buhrmester et al., 20I I). However, the fact remains that our samples typically remain more Western, Educated, Industrialized, Rich, and Democratic (Henrich et al., 20l0) than most of the world. For this reason, it would be interesting for future cross-cultural work to consider the relationship between political ideology, moralization, and free will in different cultures.

\section{Implications and Directions for Future Research}

The findings reported in this paper have theoretical implications for both the psychology of free will belief and political psychology. First, these findings provide further and more direct support for previous work conducted on free will belief as motivated social cognition (Clark, Baumeister, et al., 20I7; Clark et al., 20I4; Clark, Shniderman, et al., 20I8; Clark, Winegard, \& Baumeister, 2019; Vonasch et al., 2017). The work reported here demonstrates that belief in free will is linked to a desire to hold people accountable for their moral wrongdoing, and that free will attributions vary as a function of the valence of the action, how moral or immoral it is perceived to be, and even who the target is. Pragmatically, this highlights the fact that when exploring free will attributions, it is essential to consider - and control for - the valence and perceived morality of the event. Second, these findings provide further support for the idea that attributions of free will are malleable and context-dependent, not stable across situations and context (Bargh \& Earp, 2009). Third, these results help to shed light on the pervasive political discourse concerning responsibility and assignment of blame. The findings suggest that the emphasis within conservative political ideology on personal responsibility is directly linked to perceptions of immorality. For example, as illegal drug use is perceived to be more morally wrong, so too are drug users seen as more responsible and in control of their situation; and as being unemployed and receiving social welfare is seen to be more morally wrong, so too are people in such situations seen as being more responsible for and in control of their lot. The reason that political debates concerning responsibility and deservingness have been an enduring feature of political discourse throughout history is likely to stem, at least in part, from the powerful and often conflicting moral intuitions driving such judgments.

Following from this view, our findings have interesting theoretical and practical implications concerning the structure of policies related to behaviors that conservatives and liberals perceive as being differentially blameworthy. Consider, for example, attitudes towards unemployment benefits. The results presented here suggest that conservative policies opposing greater benefit payments are likely to be linked to the perception that hard work is a moral principle - and that not working is therefore blameworthy. Indeed, such an analysis is consistent with statements from prominent conservative leaders such as Margaret Thatcher (198I), who described her policies as "based not on some economics theory, but on things I and millions like me were brought up with [such as] an honest day's work for an honest day's pay." Political policies, it seems, are intimately tied to perceptions of morality of the actions 
concerned. This may help account for the seeming intractability of political conflict over economic policy: it is likely easier to reconcile deliberative, fact-based disagreement about the specific outcomes of policies, than it is to reconcile affect-based intuitions about moral responsibility. Put simply, while disagreement about economic outcomes can be resolved with better data, it is difficult to see an easy way to reconcile disagreements about who is morally responsible, and for what. We have no illusions that this will be easy, but our findings do suggest that political consensus on hot-topic issues such as welfare and benefits, when it can be reached, is likely to occur not through extended discussion of the economic features of the policies, but rather in achieving common moral ground. 


\section{References}

Alicke, M. (1992). Culpable causation. Journal of Personality and Social Psychology, 63(3), 368378. https://doi.org//0.1037/0022-35/4.63.3.368

Alicke, M. (2000). Culpable control and the psychology of blame. Psychological Bulletin, I26(4), 556-574. https://doi.org//0.1037/0033-2909.126.4.556

Alicke, M., Buckingham, J., Zell, E., \& Davis, T. (2008). Culpable control and counterfactual reasoning in the psychology of blame. Personality and Social Psychology Bulletin, 34(10), |37|-|38|. https://doi.org/|0. I 177/0|46|67208321594

Alicke, M., Rose, D., \& Bloom, D. (20I I). Causation, norm violation, and culpable control. The Journal of Philosophy, I08(I 2), 670-696. https://doi.org/I0.5840/jphil20 I I I08I 238

Amodio, D. M., Jost, J. T., Master, S. L., \& Yee, C. M. (2007). Neurocognitive correlates of liberalism and conservatism. Nature Neuroscience, I0(10), I246-1247. https://doi.org/10.1038/nn 1979

Aquino, K., \& Reed II, A. (2002). The self-importance of moral identity. Journal of Personality and Social Psychology, 83(6), |423-1440. https://doi.org/I0.1037/0022-35 |4.83.6.| 423

Bargh, J. A., \& Earp, B. (2009). The will is caused, not "free." Dialogue: Newsletter of the Society for Personality and Social Psychology, 24, 13-15.

Baumeister, R. F., Bratslavsky, E., Finkenauer, C., \& Vohs, K. D. (200I). Bad is stronger than good. Review of General Psychology, 5(4), 323-370. https://doi.org/I0.1037/I0892680.5.4.323

Baumeister, R. F., \& Monroe, A. E. (20I4). Recent research on free will: Conceptualizations, beliefs, and processes. Advances in Experimental Social Psychology, 50, I-52.

Baumeister, R. F., \& Newman, L. S. (1994). Self-regulation of cognitive inference and decision processes. Personality and Social Psychology Bulletin, 20(I), 3-19.

https://doi.org/10.1 I77/0146167294201001

Buhrmester, M., Kwang, T., \& Gosling, S. D. (201 I). Amazon's Mechanical Turk: A new source of inexpensive, yet high-quality, data? Perspectives on Psychological Science, 6(I), 3-5. https://doi.org//0.1 I77//74569/610393980

Cameron, D. (2010, May II). David Cameron's speech in full. The Guardian. https://www.theguardian.com/politics/2010/may/I I/david-cameron-speech-full-text

Carey, J. M., \& Paulhus, D. L. (20I3). Worldview implications of believing in free will and/or determinism: Politics, morality, and punitiveness. Journal of Personality, 8 I (2), I30|4I. https://doi.org/ I0. I I I I/j. I 467-6494.20 I 2.00799.x

Carroll, J. S., Perkowitz, W. T., Lurigio, A. J., \& Weaver, F. M. (1987). Sentencing goals, causal attributions, ideology, and personality. Journal of Personality, 52(I), 107-I I8.

Cawley, M. J., Martin, J. E., \& Johnson, J. A. (2000). A virtues approach to personality. Personality and Individual Differences, 28(5), 997-1013. https://doi.org/I0.1016/S0I918869(99)00207-X

Clark, C. J., Baumeister, R. F., \& Ditto, P. H. (2017). Making punishment palatable: Belief in free will alleviates punitive distress. Consciousness and Cognition, 5I, I93-2II. https://doi.org/10.1016/j.concog.2017.03.010 
Clark, C. J., Chen, E. E., \& Ditto, P. H. (20I5). Moral coherence processes: Constructing culpability and consequences. Current Opinion in Psychology, 6, 123-128. https://doi.org/10.1016/j.copsyc.2015.07.016

Clark, C. J., Luguri, J. B., Ditto, P. H., Knobe, J., Shariff, A. F., \& Baumeister, R. F. (20l4). Free to punish: A motivated account of free will belief. Journal of Personality and Social Psychology, 106(4), 50I. https://doi.org/I0.1037/a0035880

Clark, C. J., Shniderman, A., Baumeister, R. F., Luguri, J. B., \& Ditto, P. H. (2018). Are morally good actions ever free? Consciousness and Cognition, 63, I6I-182.

Clark, C. J., Winegard, B. M., \& Baumeister, R. F. (2019). Forget the Folk: Moral Responsibility Preservation Motives and Other Conditions for Compatibilism. Frontiers in Psychology, 10. https://doi.org/10.3389/fpsyg.2019.00215

Clark, C. J., Winegard, B. M., \& Shariff, A. F. (2019). Motivated free will beliefs: The theory, new (preregistered) studies, and three meta-analyses. Manuscript Submitted for Publication.

Costa, P. T., \& McCrae, R. R. (1992a). Four ways five factors are basic. Personality and Individual Differences, 13(6), 653-665. https://doi.org/10.1016/0191-8869(92)90236-I

Costa, P. T., \& McCrae, R. R. (1992b). The Five-Factor Model of Personality and Its Relevance to Personality Disorders. Journal of Personality Disorders, 6(4), 343-359. https://doi.org/I0.152I/pedi. I 992.6.4.343

Crandall, C. S. (1994). Prejudice against fat people: Ideology and self-interest. Journal of Personality and Social Psychology, 66(5), 882-894. https://doi.org//0.1037/002235|4.66.5.882

Cushman, F., Knobe, J., \& Sinnott-Armstrong, W. (2008). Moral appraisals affect doing/allowing judgments. Cognition, 108(I), 28I-289. https://doi.org/10.1016/j.cognition.2008.02.005

Ditto, P. H., \& Boardman, A. F. (1995). Perceived accuracy of favorable and unfavorable psychological feedback. Basic and Applied Social Psychology, I6(I-2), I37-I57. https://doi.org/ I0.1207/s I5324834basp I60 I\&2_9

Ditto, P. H., \& Lopez, D. F. (1992). Motivated skepticism: Use of differential decision criteria for preferred and nonpreferred conclusions. Journal of Personality and Social Psychology, 63(4), 568-584. https://doi.org/I0.1037/0022-35I4.63.4.568

Ditto, P. H., Pizarro, D. A., \& Tannenbaum, D. (2009). Motivated moral reasoning. Psychology of Learning and Motivation, 50, 307-338. https://doi.org/10.1016/S0079-

742 I (08)004l 0-6

Dunning, D., Leuenberger, A., \& Sherman, D. A. (1995). A new look at motivated inference: Are self-serving theories of success a product of motivational forces? Journal of Personality and Social Psychology, 69(I), 58-68. https://doi.org/I0.1037/002235I4.69.1.58

Eidelman, S., Crandall, C. S., Goodman, J. A., \& Blanchar, J. C. (2012). Low-effort thought promotes political conservatism. Personality and Social Psychology Bulletin, 38(6), 808820. https://doi.org/10.1 177/0146167212439213 
Feather, N. T. (1985). Attitudes, values, and attributions: Explanations of unemployment. Journal of Personality and Social Psychology, 48(4), 876-889. https://doi.org/| 0.1037/0022-35 |4.48.4.876

Feldman, G., Wong, K. F. E., \& Baumeister, R. F. (2016). Bad is freer than good: Positivenegative asymmetry in attributions of free will. Consciousness and Cognition, 42, 26-40. https://doi.org/10.1016/j.concog.2016.03.005

Frimer, J. A., Tell, C. E., \& Motyl, M. (2017). Sacralizing liberals and fair-minded conservatives: Ideological symmetry in the moral motives in the culture war. Analyses of Social Issues and Public Policy, I 7(I), 33-59. https://doi.org/ I0. I I I I/asap. I 2 I 27

Goh, J. X., Hall, J. A., \& Rosenthal, R. (2016). Mini meta-analysis of your own studies: Some arguments on why and a primer on how. Social and Personality Psychology Compass, I0(10), 535-549. https://doi.org//0.1 I I I/spc3.12267

Graham, J., \& Haidt, J. (2012). Sacred values and evil adversaries: A moral foundations approach. In The social psychology of morality: Exploring the causes of good and evil (pp. II-3I). American Psychological Association. https://doi.org/I0.1037/I309I-00 I

Graham, J., Haidt, J., Koleva, S., Motyl, M., lyer, R., Wojcik, S. P., \& Ditto, P. H. (20I3). Moral foundations theory: The pragmatic validity of moral pluralism. Advances in Experimental Social Psychology, 47, 55-130.

Graham, J., Haidt, J., \& Nosek, B. A. (2009). Liberals and conservatives rely on different sets of moral foundations. Journal of Personality and Social Psychology, 96(5), 1029. https://doi.org/|0.1037/a0015141

Graham, J., Nosek, B. A., Haidt, J., lyer, R., Koleva, S., \& Ditto, P. H. (20I I). Mapping the moral domain. Journal of Personality and Social Psychology, I0I(2), 366-385. https://doi.org// 0.1037/a002/847

Haidt, J. (200I). The emotional dog and its rational tail: A social intuitionist approach to moral judgment. Psychological Review, I08(4), 8|4-834. https://doi.org/I0.1037/0033295X.108.4.8I4

Haidt, J. (2012). The righteous mind: Why good people are divided by politics and religion. Vintage. Hamlin, J. K., \& Baron, A. S. (20I4). Agency attribution in infancy: Evidence for a negativity bias. PLOS ONE, 9(5), e96I I2. https://doi.org/ I0. I37//journal.pone.0096 I I 2

Harris, L. T., \& Fiske, S. T. (2006). Dehumanizing the lowest of the low: Neuroimaging responses to extreme out-groups. Psychological Science, I7(10), 847-853. https://doi.org/10.1III/j. I467-9280.2006.01793.x

Hatemi, P. K., Crabtree, C., \& Smith, K. B. (2019). Ideology justifies morality: Political beliefs predict moral foundations. American Journal of Political Science, 63(4), 788-806. https://doi.org/ I 0. I I I I/ajps. I 2448

Hatemi, P. K., Medland, S. E., Klemmensen, R., Oskarrson, S., Littvay, L., Dawes, C., Verhulst, B., McDermott, R., Nørgaard, A. S., Klofstad, C., Christensen, K., Johannesson, M., Magnusson, P. K. E., Eaves, L. J., \& Martin, N. G. (20I4). Genetic influences on political ideologies: Twin analyses of 19 measures of political ideologies from five democracies and genome-wide findings from three populations. Behavior Genetics, 44(3), 282-294. https://doi.org/10.1007/s I0519-0I4-9648-8 
Hedges, L., \& Vevea, J. (1998). Fixed- and random-effects models in meta-analysis.

Psychological Methods, 3(4), 486-504.

Henrich, J., Heine, S. J., \& Norenzayan, A. (2010). The weirdest people in the world?

Behavioral and Brain Sciences, 33(2-3), 6I-83.

https://doi.org/10.1017/S0140525X0999152X

Jost, J. T. (2006). The end of the end of ideology. American Psychologist, 6I (7), 65 I-670. https://doi.org/ I0.1037/0003-066X.61.7.65 I

Jost, J. T., Federico, C. M., \& Napier, J. L. (2009). Political ideology: Its structure, functions, and elective affinities. Annual Review of Psychology, 60(I), 307-337. https://doi.org/10.1 I46/annurev.psych.60.1 I0707.163600

Jost, J. T., Nosek, B. A., \& Gosling, S. D. (2008). Ideology: Its resurgence in social, personality, and political psychology. Perspectives on Psychological Science, 3(2), I26| 36. https://doi.org/ | 0. I I I I/j. I745-69 |6.2008.00070.x

Knobe, J. (2003). Intentional action and side effects in ordinary language. Analysis, 63(279), 190-194. https://doi.org/ I0.1 I I I/|467-8284.00419

Knobe, J., \& Fraser, B. (2008). Causal judgment and moral judgment: Two experiments. In W. Sinnott-Armstrong (Ed.), Moral Psychology. MIT Press.

Kugler, M., Jost, J. T., \& Noorbaloochi, S. (20I4). Another look at moral foundations theory: Do authoritarianism and social dominance orientation explain liberal-conservative differences in "moral” intuitions? Social Justice Research, 27(4), 4I3-43I. https://doi.org/ | 0.1007/s | |2 I I-0 |4-0223-5

Lakens, D. (20I4). Performing high-powered studies efficiently with sequential analyses. European Journal of Social Psychology, 44(7), 701-710.

Lapsley, D. K., \& Lasky, B. (200I). Prototypic Moral Character. Identity, I (4), 345-363. https://doi.org/I0.1207/SI532706XID0I04_03

Leslie, A. M., Knobe, J., \& Cohen, A. (2006). Acting intentionally and the side-effect effect: Theory of mind and moral judgment. Psychological Science, I 7(5), 42I-427. https://doi.org/ I0. I I I I/j. I467-9280.2006.01722.x

Lovett, B. J., Jordan, A. H., \& Wiltermuth, S. S. (20I2). Individual differences in the moralization of everyday life. Ethics \& Behavior, 22(4), 248-257. https://doi.org/10.1080/10508422.2012.659132

McAdams, D. P., Albaugh, M., Farber, E., Daniels, J., Logan, R. L., \& Olson, B. (2008). Family metaphors and moral intuitions: How conservatives and liberals narrate their lives. Journal of Personality and Social Psychology, 95(4), 978-990. https://doi.org/I0.1037/a001 2650

Monroe, A. E., \& Ysidron, D. (2019). Not so motivated after all? Three replication attempts and a theoretical challenge to a morally-motivated belief in free will. Manuscript Submitted for Publication.

Mooney, C. (20I2). The Republican brain: The science of why they deny science-and reality. John Wiley \& Sons.

Newman, L. S., Tan, M., Caldwell, T. L., Duff, K. J., \& Winer, E. S. (20I8). Name norms: A guide to casting your next experiment. Personality and Social Psychology Bulletin,

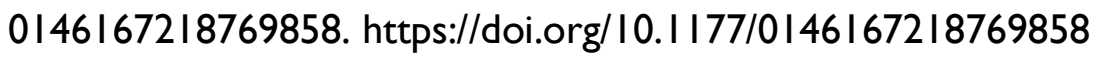


Nichols, S. (2007). The rise of compatibilism: A case study in the quantitative history of philosophy. Midwest Studies In Philosophy, 3I (I), 260-270. https://doi.org/I0. I I I I/j. I475-4975.2007.00I52.x

Nichols, S., \& Knobe, J. (2007). Moral responsibility and determinism: The cognitive science of folk intuitions. Noûs, 4I (4), 663-685. https://doi.org//0.1 I I //j. I4680068.2007.00666.x

Padilla-Walker, L. M., \& Jensen, L. A. (2016). Validation of the long- and short-form of the Ethical Values Assessment (EVA): A questionnaire measuring the three ethics approach to moral psychology. International Journal of Behavioral Development, 40(2), I8I-192. https://doi.org/ |0.I |77/0 |650254|5587534

Paolacci, G., \& Chandler, J. (20I4). Inside the Turk: Understanding Mechanical Turk as a Participant Pool. Current Directions in Psychological Science, 23(3), 184-188. https://doi.org//0.1I77/096372/414531598

Paulhus, D. L., \& Carey, J. M. (20I I). The FAD-Plus: Measuring Lay Beliefs Regarding Free Will and Related Constructs. Journal of Personality Assessment, 93(1), 96-104. https://doi.org/ 10.1080/00223891.2010.528483

Paulhus, D. L., \& Margesson, A. (1994). Free Will and Scientific Determinism (FAD-4) scale. Unpublished instrument, University of British Columbia, Vancouver, BC, Canada.

Pettit, D., \& Knobe, J. (2009). The pervasive impact of moral judgment. Mind \& Language, 24(5), 586-604. https://doi.org/I0.I I I I/j. I468-00 I7.2009.0I375.x

Phillips, J., \& Knobe, J. (2009). Moral judgments and intuitions about freedom. Psychological Inquiry, 20(I), 30-36. https://doi.org/10.1080/I0478400902744279

Reagan, R. ( I 968, July 3 I). Excerpts of a Speech by Governor Ronald Reagan. Republican National Convention, Miama, FL. https://en.wikiquote.org/wiki/Ronald_Reagan\#1960s

Sagarin, B. J., Ambler, J. K., \& Lee, E. M. (20I4). An Ethical Approach to Peeking at Data: Perspectives on Psychological Science. https://doi.org/I0.1 I77/174569/6145282 I4

Sargent, M. J. (2004). Less Thought, More Punishment: Need for Cognition Predicts Support for Punitive Responses to Crime. Personality and Social Psychology Bulletin, 30(I I), |485-|493. https://doi.org/|0. I | 77/0| 46 |6720426448 |

Skitka, L. J., Mullen, E., Griffin, T., Hutchinson, S., \& Chamberlin, B. (2002). Dispositions, scripts, or motivated correction?: Understanding ideological differences in explanations for social problems. Journal of Personality and Social Psychology, 83(2), 470-487. https://doi.org//0.1037/0022-35/4.83.2.470

Skitka, L. J., \& Tetlock, P. E. (1992). Allocating scarce resources: A contingency model of distributive justice. Journal of Experimental Social Psychology, 28(6), 49I-522. https://doi.org/I0.1016/0022-1031(92)90043-J

Skitka, L. J., \& Tetlock, P. E. (1993). Providing public assistance: Cognitive and motivational processes underlying liberal and conservative policy preferences. Journal of Personality and Social Psychology, 65(6), I205. https://doi.org/I0.1037/0022-35|4.65.6.1205

Smith, K. B., Alford, J. R., Hibbing, J. R., Martin, N. G., \& Hatemi, P. K. (20I7). Intuitive ethics and political orientations: Testing moral foundations as a theory of political ideology. American Journal of Political Science, 6 I (2), 424-437. https://doi.org/ I 0. I I I I/ajps. 12255 
Smith, K. D., Smith, S. T., \& Christopher, J. C. (2007). What defines the good person? Cross-cultural comparisons of experts' models with lay prototypes. Journal of CrossCultural Psychology, 38(3), 333-360. https://doi.org/I 0.I I77/0022022I 07300279

Thatcher, M. (198I, September 20). My policies are based not on some economics theory... The News of The World. https://en.wikiquote.org/wiki/Margaret_Thatcher

Vonasch, A. J., Clark, C. J., Lau, S., Vohs, K. D., \& Baumeister, R. F. (2017). Ordinary people associate addiction with loss of free will. Addictive Behaviors Reports, 5, 56-66. https://doi.org/10.1016/j.abrep.2017.01.002

Walker, L. J., \& Pitts, R. C. (1998). Naturalistic conceptions of moral maturity. Developmental Psychology, 34(3), 403-4I9.

Zucker, G. S., \& Weiner, B. (1993). Conservatism and perceptions of poverty: An attributional analysis. Journal of Applied Social Psychology, 23(I 2), 925-943. https://doi.org/I0.1 III/j.1559-1816.1993.tb01014.x 